\title{
Stochastic CGL equations without linear dispersion in any space dimension
}

\author{
Sergei Kuksin • Vahagn Nersesyan
}

Received: 5 August 2012 / Published online: 17 May 2013

(C) Springer Science+Business Media New York 2013

Abstract We consider the stochastic CGL equation

$$
\dot{u}-v \Delta u+(i+a)|u|^{2} u=\eta(t, x), \quad \operatorname{dim} x=n,
$$

where $v>0$ and $a \geq 0$, in a cube (or in a smooth bounded domain) with Dirichlet boundary condition. The force $\eta$ is white in time, regular in $x$ and non-degenerate. We study this equation in the space of continuous complex functions $u(x)$, and prove that for any $n$ it defines there a unique mixing Markov process. So for a large class of functionals $f(u(\cdot))$ and for any solution $u(t, x)$, the averaged observable $\mathbb{E} f(u(t, \cdot))$ converges to a quantity, independent from the initial data $u(0, x)$, and equal to the integral of $f(u)$ against the unique stationary measure of the equation.

Keywords Complex Ginzburg-Landau equation $\cdot$ Random force $\cdot$ Mixing $\cdot$ Markov process

\section{Introduction}

We study the stochastic CGL equation

$$
\dot{u}-v \Delta u+(i+a)|u|^{2} u=\eta(t, x), \quad \operatorname{dim} x=n,
$$

\footnotetext{
S. Kuksin $(\varangle)$

CNRS and IMJ, Paris 7, 175 rue du Chevaleret, 75013 Paris, France

e-mail:kuksin@gmail.com

V. Nersesyan

Laboratoire de Mathématiques, UMR CNRS 8100,

Université de Versailles-Saint-Quentin-en-Yvelines, 78035 Versailles, France

e-mail: Vahagn.Nersesyan@math.uvsq.fr
} 
where $n$ is any, $v>0, a \geq 0$ and the random force $\eta$ is white in time and regular in $x$. All our results and constructions are uniform in $a$ from bounded intervals $[0, C], C \geq 0$. Since for $a>0$ the equation possesses extra properties due to the nonlinear dissipation (it is "stabler"), then below we restrict ourselves to the more complicated case $a=0$; see discussion in Sect. 5. This equation is the Hamiltonian system $\dot{u}+i|u|^{2} u=0$, damped by the viscous term $v \Delta u$ and driven by the random force $\eta$. So it makes a model for the stochastic Navier-Stokes system, which may be regarded as a damped-driven Euler equation (which is a Hamiltonian system, homogeneous of degree two). In this work we are not concerned with the interesting turbulence-limit $v \rightarrow 0$ (see $[15,16]$ for some related results) and, again to simplify notation, choose $v=1$. That is, we consider the equation

$$
\dot{u}-\Delta u+i|u|^{2} u=\eta(t, x) .
$$

For the space-domain we take the cube $K=[0, \pi]^{n}$ with the Dirichlet boundary conditions, which we regard as the odd periodic boundary conditions

$$
u\left(t, \ldots, x_{j}, \ldots\right)=u\left(t, \ldots, x_{j}+2 \pi, \ldots\right)=-u\left(t, \ldots,-x_{j}, \ldots\right) \quad \forall j .
$$

Our results remain true for (1.2) in a smooth bounded domain with the Dirichlet boundary conditions, see Sect. 5 .

The force $\eta(t, x)$ is a random field of the form

$$
\eta(t, x)=\frac{\partial}{\partial t} \zeta(t, x), \quad \zeta(t, x)=\sum_{d \in \mathbb{N}^{n}} b_{d} \beta_{d}(t) \varphi_{d}(x) .
$$

Here $b_{d}$ are real numbers such that

$$
B_{*}:=\sum_{d \in \mathbb{N}^{n}}\left|b_{d}\right|<\infty
$$

$\beta_{d}=\beta_{d}^{R}+i \beta_{d}^{I}$, where $\beta_{d}^{R}, \beta_{d}^{I}$ are standard independent (real-valued) Brownian motions, defined on a complete probability space $(\Omega, \mathcal{F}, \mathbb{P})$ with a filtration $\left\{\mathcal{F}_{t} ; t \geq 0\right\} .^{1}$ The set of real functions $\left\{\varphi_{d}(x), d \in \mathbb{N}^{n}\right\}$ is the $L^{2}$-normalised system of eigenfunctions of the Laplacian,

$$
\varphi_{d}(x)=(2 / \pi)^{n / 2} \sin \left(d_{1} x_{1}\right) \cdot \ldots \cdot \sin \left(d_{n} x_{n}\right), \quad(-\Delta) \varphi_{d}=\alpha_{d} \varphi_{d}, \quad \alpha_{d}=|d|^{2} .
$$

Since we impose no restriction on the dimension $n$, then global solvability of Eq. (1.2) cannot be established using the $L_{2}$-Sobolev spaces. Moreover, as the best a priori estimates, available for its solutions, turned out to be in terms of the $L_{\infty}$-norm, then the methods, developed to treat stochastic PDE in reflexive Banach spaces (e.g., see [1,7]) also are not applicable to (1.2). Instead we take the approach of the work [16] which

\footnotetext{
1 The filtered probability space $\left(\Omega, \mathcal{F},\left\{\mathcal{F}_{t}\right\}, \mathbb{P}\right)$, as well as all other filtered probability spaces, used in this work, are assumed to satisfy the usual condition, see Definition 2.29 in [12].
} 
exploits essentially the well known fact that the deterministic equation (1.2 $)_{\eta=0}$ implies for the real function $|u(t, x)|$ a parabolic inequality with the maximum principle.

Denote by $H^{m}$ the Sobolev space of order $m$, formed by complex odd periodic functions and given the norm

$$
\|u\|_{m}=\left\|(-\Delta)^{m / 2} u\right\|,
$$

where $\|\cdot\|$ is the $L^{2}$-norm on the cube $K$. In Sect. 2.1 we repeat some construction from [16] and state its main result, which says that if

$$
u(0, x)=u_{0}(x)
$$

where $u_{0} \in H^{m}, m>n / 2$, and

$$
B_{m}:=\sum_{d} b_{d}^{2}|d|^{2 m}<\infty,
$$

then (1.2), (1.6) has a unique strong solution $u(t) \in H^{m}$. Moreover, for any $T \geq 0$ the random variable $X_{T}=\sup _{T \leq t \leq T+1}|u(t)|_{\infty}^{2}$ satisfies the estimates

$$
\mathbb{E} X_{T}^{q} \leq C_{q} \quad \forall q \geq 0
$$

where $C_{q}$ depends only on $\left|u_{0}\right|_{\infty}$ and $B_{*}$. Analysis of the constants $C_{q}$, made in Sect. 2.2, implies that suitable exponential moments of the variables $X_{T}$ are finite:

$$
\mathbb{E} e^{c X_{T}} \leq C^{\prime}=C^{\prime}\left(B_{*},\left|u_{0}\right|_{\infty}\right),
$$

where $c>0$ depends only on $B_{*}$.

Denote by $C_{0}(K)$ the space of continuous complex functions on $K$, vanishing at $\partial K$. In Sect. 3 we consider the initial-value problem (1.2), (1.6), assuming only that $B_{*}<\infty$ and $u_{0} \in C_{0}(K)$. Approximating it by the regular problems as above and using that the constants in (1.8), (1.9) depend only on $B_{*}$ and $\left|u_{0}\right|_{\infty}$, we prove

Theorem 1.1 Let $B_{*}<\infty$ and $u_{0} \in C_{0}(K)$. Then the problem (1.2), (1.6) has a unique strong solution $u(t, x)$ which almost surely belongs to the space $C\left([0, \infty), C_{0}(K)\right) \cap L_{\text {loc }}^{2}\left([0, \infty), H^{1}\right)$. The solutions $u$ define in the space $C_{0}(K) a$ Fellerian Markov process.

Consider the quantities $J^{t}=\int_{0}^{t}|u(\tau)|_{\infty}^{2} d \tau-K t$, where $K$ is a suitable constant, depending only on $B_{*}$. Based on (1.9), we prove in Lemma 2.8 that the random variable $\sup _{t \geq 0} J^{t}$ has exponentially bounded tails. Since the non-autonomous term in the linearised equation (1.2) is quadratic in $u, \bar{u}$, then the method to treat the $2 \mathrm{~d}$ stochastic Navier-Stokes system, based on the Foias-Prodi estimate and the Girsanov theorem (see [14] for discussion and references to the original works) allows us to prove in Sect. 4

(stability) There is a constant $L \geq 1$ and two sequences $\left\{T_{m} \geq 0, m \geq 1\right\}$ and $\left\{\varepsilon_{m}>0, m \geq 1\right\}, \varepsilon_{m} \rightarrow 0$ as $m \rightarrow \infty$, such that if for any $m \geq 1$ solutions $u(t)$ and $u^{\prime}(t)$ of (1.2) satisfy 


$$
u(0), u^{\prime}(0) \in G_{m}=\left\{u \in C_{0}(K):\|u\| \leq 1 / m,|u|_{L_{\infty}} \leq L\right\},
$$

then for each $t \geq T_{m}$ we have $\left\|\mathcal{D}(u(t))-\mathcal{D}\left(u^{\prime}(t)\right)\right\|_{\mathcal{L}}^{*} \leq \varepsilon_{m}$. Here $\|\mu-v\|_{\mathcal{L}}^{*}$ is the dual-Lipschitz distance between Borelian measures $\mu$ and $v$ on the space $H^{0}$ (see below Notation).

We also verify in Sect. 4 that

(recurrence) For each $m \geq 1$ and for any $u_{0}, u_{0}^{\prime} \in C_{0}(K)$, the hitting time inf $\{t \geq$ $\left.0: u(t) \in G_{m}, u^{\prime}(t) \in G_{m}\right\}$, where $u(t)$ and $u^{\prime}(t)$ are two independent solutions of (1.2) such that $u(0)=u_{0}$ and $u^{\prime}(0)=u_{0}^{\prime}$, is almost surely finite.

These two properties allow us to use Theorem 3.1.3 from [14]. ${ }^{2}$ That result provides the weakest known sufficient condition to guarantee the mixing in the random system, corresponding to a stochastic PDE. It applies to systems in Banach spaces, assuming that the random force $\eta$ is non degenerate (in the sense that its sufficiently many Fourier coefficients are non-zero), and does not imply the exponential mixing. We note that there are other theorems which, under stronger assumptions on a system, claim the exponential mixing (see Theorem 3.1.7 in [14] and discussion in that book); some of them apply to systems in Hilbert spaces with degenerate random forces, see [9]. The application of Theorem 3.1.3 from [14] implies the second main result of this work:

Theorem 1.2 There is an integer $N=N\left(B_{*}, v\right) \geq 1$ such that if $b_{d} \neq 0$ for $|d| \leq N$, then the Markov process, constructed in Theorem 1.1, is mixing. That is, it has a unique stationary measure $\mu$, and every solution $u(t)$ converges to $\mu$ in distribution.

This theorem implies that for any continuous functional $f$ on $C_{0}(K)$ such that $|f(u)| \leq$ $C e^{c|u|_{\infty}^{2}}$ we have the convergence

$$
\mathbb{E} f(u(t)) \rightarrow \int f(v) \mu(d v) \text { as } t \rightarrow \infty,
$$

where $u(t)$ is any solution of (1.2). See Corollary 4.3.

In Sect. 5 we explain that our results also apply to equations (1.1), considered in smooth bounded domains in $\mathbb{R}^{n}$ with Dirichlet boundary conditions; that Theorem 1.1 generalises to equations

$$
\dot{u}-v \Delta u+(i+a) g_{r}\left(|u|^{2}\right) u=\eta(t, x),
$$

where $g_{r}(t)$ is a smooth function, equal to $t^{r}, r \geq 0$, for $t \geq 1$, and Theorem 1.2 generalises to Eq. (1.10) with $0 \leq r \leq 1$.

Similar results for the CGL equations (1.10), where $\eta$ is a kick force, hold without the restriction that the nonlinearity is cubic, see in [14]. Same is true when $\eta$ is the derivative of a compound Poisson process, see [20].

Our technique does not apply to equations (1.10) with complex $v$. To prove analogies of Theorems 1.1, 1.2 for such equations, strong restrictions should be imposed on $n$ and $r$. See [8,21] for equations with $\operatorname{Re} v>0$ and $a>0$, and see [23] for the case

\footnotetext{
2 That result was introduced in [23], based on ideas, developed in [13] to establish mixing for the stochastic 2D NSE.
} 
$\operatorname{Re} v>0$ and $a=0$. We also mention the work [4] which treats interesting class of one-dimensional equations (1.1) with complex $v$ such that $\operatorname{Re} v=0$ and $a=0$, damped by the term $\alpha u$ in the l.h.s. of the equation.

Notation By $H$ we denote the $L^{2}$-space of odd $2 \pi$-periodic complex functions with the scalar product $\langle u, v\rangle:=\operatorname{Re} \int_{K} u(x) \bar{v}(x) \mathrm{d} x$ and the norm $\|u\|^{2}:=\langle u, u\rangle$; by $H^{m}(K), m \geq 0$ - the Sobolev space of odd $2 \pi$-periodic complex functions of order $m$, endowed with the homogeneous norm $(1.5)\left(\operatorname{so} H^{0}(K)=H\right.$ and $\left.\|\cdot\|_{0}=\|\cdot\|\right)$. By $C_{0}(Q)$ we denote the space of continuous complex functions on a closed domain $Q$ which vanish at the boundary $\partial Q$ (note that the space $C_{0}(K)$ is formed by restrictions to $K$ of continuous odd periodic functions).

For a Banach space $X$ we denote:

$C_{b}(X)$ - the space of real-valued bounded continuous functions on $X$;

$\mathcal{L}(X)$ - the space of bounded Lipschitz functions $f$ on $X$, given the norm

$$
\|f\|_{\mathcal{L}}:=|f|_{\infty}+\operatorname{Lip}(f)<\infty, \quad \operatorname{Lip}(f):=\sup _{u \neq v}|f(u)-f(v)|\|u-v\|^{-1}
$$

$\mathcal{B}(X)$-the $\sigma$-algebra of Borel subsets of $X$;

$\mathcal{P}(X)$ - the set of probability measures on $(X, \mathcal{B}(X))$;

$B_{X}(d), d>0$ - the open ball in $X$ of radius $d$, centered at the origin.

For $\mu \in \mathcal{P}(X)$ and $f \in C_{b}(X)$ we denote $(f, \mu)=(\mu, f)=\int_{X} f(u) \mu(\mathrm{d} u)$. If $\mu_{1}, \mu_{2} \in \mathcal{P}(X)$, we set

$$
\begin{aligned}
\left\|\mu_{1}-\mu_{2}\right\|_{\mathcal{L}}^{*} & =\sup \left\{\left|\left(f, \mu_{1}\right)-\left(f, \mu_{2}\right)\right|: f \in \mathcal{L}(X),\|f\|_{\mathcal{L}} \leq 1\right\}, \\
\left\|\mu_{1}-\mu_{2}\right\|_{\text {var }} & =\sup \left\{\left|\mu_{1}(\Gamma)-\mu_{2}(\Gamma)\right|: \Gamma \in \mathcal{B}(X)\right\}
\end{aligned}
$$

The arrow $\rightarrow$ indicates the weak convergence of measures in $\mathcal{P}(X)$. It is well known that $\mu_{n} \rightarrow \mu$ if and only if $\left\|\mu_{n}-\mu\right\|_{\mathcal{L}}^{*} \rightarrow 0$, and that $\left\|\mu_{1}-\mu_{2}\right\|_{\mathcal{L}}^{*} \leq 2\left\|\mu_{1}-\mu_{2}\right\|_{\text {var }}$.

The distribution of a random variable $\xi$ is denoted by $\mathcal{D}(\xi)$. For complex numbers $z_{1}, z_{2}$ we denote $z_{1} \cdot z_{2}=\operatorname{Re} z_{1} \bar{z}_{2} ;$ so $z \cdot \mathrm{d} \beta_{d}=(\operatorname{Re} z) \mathrm{d} \beta_{d}^{R}+(\operatorname{Im} z) \mathrm{d} \beta_{d}^{I}$. We denote by $C, C_{1}$ etc. unessential positive constants.

\section{Stochastic CGL equation}

\subsection{Strong and weak solutions}

Let the filtered probability space $\left(\Omega, \mathcal{F},\left\{\mathcal{F}_{t}\right\}, \mathbb{P}\right)$ be as in Introduction. We use the standard definitions of strong and weak solutions for stochastic PDEs (e.g., see [12]):

Definition 2.1 Let $0<T<\infty$. A random process $u(t)=u(t, x), t \in[0, T]$ in $C_{0}(K)$ defined on a probability space $(\Omega, \mathcal{F}, \mathbb{P})$ is called a strong solution of (1.2), (1.6) if the following three conditions hold:

(i) the process $u(t)$ is adapted to the filtration $\mathcal{F}_{t}$; 
(ii) its trajectories $u(t)$ a.s. belong to the space

$$
\mathcal{H}([0, T]):=C\left([0, T], C_{0}(K)\right) \cap L^{2}\left([0, T], H^{1}\right)
$$

(iii) for every $t \in[0, T]$ a.s. we have

$$
u(t)=u_{0}+\int_{0}^{t}\left(\Delta u-i|u|^{2} u\right) \mathrm{d} s+\zeta(t)
$$

where both sides are regarded as elements of $H^{-1}$.

If (i)-(iii) hold for every $T<\infty$, then $u(t)$ is called a strong solution for $t \in \mathbb{R}_{+}=$ $[0, \infty)$.

A continuous adapted process $u(t) \in C_{0}(K)$ and a Wiener process $\zeta^{\prime}(t) \in H$, defined in some filtered probability space, are called a weak solution of (1.2) if $\mathcal{D}\left(\zeta^{\prime}\right)=$ $\mathcal{D}(\zeta)$ and (ii), (iii) of Definition 2.1 hold with $\zeta$ replaced by $\zeta^{\prime}$.

Recalling notation (1.7), we note that $B_{0} \leq B_{*}^{2}<\infty$. Let us fix any

$$
m>n / 2
$$

Problem (1.2), (1.6) with $u_{0} \in H^{m}$ and $B_{m}<+\infty$ was considered in [16]. Choosing $\delta=1$ in [16], we state Theorem 4 of that work as follows:

Theorem 2.2 Assume that $u_{0} \in H^{m}$ and $B_{m}<+\infty$. Then (1.2), (1.6) has a unique strong solution $u$ which is in $\mathcal{H}([0, \infty))$ a.s., and for any $t \geq 0, q \geq 1$ satisfies the estimates

$$
\begin{aligned}
\mathbb{E} \sup _{s \in[t, t+1]}|u(s)|_{\infty}^{q} & \leq C_{q}, \\
\mathbb{E}\|u(t)\|_{m}^{q} & \leq C_{q, m},
\end{aligned}
$$

where $C_{q}$ is a constant depending on $\left|u_{0}\right|_{\infty}$, while $C_{q, m}$ also depends on $\left\|u_{0}\right\|_{m}$ and $B_{m}$.

In this theorem and everywhere below the constants depend on $n$ and $B_{*}$. We do not indicate this dependence.

Remark 2.3 It was assumed in [16] that $n \leq 3$. This assumption is not needed for the proof. The force $\eta(t, x)$ in [16] has the form $\eta(t, x) \dot{\beta}(t)$, where $\beta$ is the standard Brownian motion and $\eta(t, x)$ is a random field, continuous and bounded uniformly in $(t, x)$, smooth in $x$ and progressively measurable. The proof without any change applies to forces of the form (1.3).

Our next goal is to get more estimates for solutions $u(t, x)$. Applying Itô's formula to $\|u\|^{2}$, where $u(t)=\sum u_{d}(t) \varphi_{d}(x)$ is a solution constructed in Theorem 2.2, we find that 


$$
\|u(t)\|^{2}=\left\|u_{0}\right\|^{2}+\int_{0}^{t}\left(-2\|u(\tau)\|_{1}^{2}+2 B_{0}\right) \mathrm{d} \tau+2 \sum_{d \in \mathbb{N} n} b_{d} \int_{0}^{t} u_{d}(\tau) \cdot \mathrm{d} \beta_{d}(\tau) .
$$

Taking the expectation, we get for any $t \geq 0$

$$
\mathbb{E}\|u(t)\|^{2}+2 \mathbb{E} \int_{0}^{t}\|u(\tau)\|_{1}^{2} \mathrm{~d} \tau=\left\|u_{0}\right\|^{2}+2 B_{0} t
$$

To get more involved estimates, we first repeat a construction from [16] which evokes the maximum principle to bound the norm $|u(t, x)|$ of a solution $u(t, x)$ as in Theorem 2.2 in terms of a solution of a stochastic heat equation.

Let $\xi \in C^{\infty}(\mathbb{R})$ be any function such that

$$
\xi(r)= \begin{cases}0 & \text { for } r \leq \frac{1}{4} \\ r & \text { for } r \geq \frac{1}{2}\end{cases}
$$

Writing $u$ in the polar form $u=r e^{i \phi}$ and using the Itô formula for $\xi(|u|)$ (see [6], Section 4.5 and [14], Section 7.7), we get

$$
\begin{aligned}
\xi(r)= & \xi_{0}+\int_{0}^{t}\left[\xi^{\prime}(r)\left(\Delta r-r|\nabla \phi|^{2}\right)+\frac{1}{2} \sum_{d \in \mathbb{N}^{n}} b_{d}^{2}\left(\xi^{\prime \prime}(r)\left(e^{i \phi} \cdot \varphi_{d}\right)^{2}\right.\right. \\
& \left.\left.+\xi^{\prime}(r) \frac{1}{r}\left(\left|\varphi_{d}\right|^{2}-\left(e^{i \phi} \cdot \varphi_{d}\right)^{2}\right)\right)\right] \mathrm{d} t+\Upsilon(t),
\end{aligned}
$$

where $\xi_{0}=\xi\left(\left|u_{0}\right|\right), a \cdot b=\operatorname{Re} a \bar{b}$ for $a, b \in \mathbb{C}$ and $\Upsilon(t)$ is the real Wiener process

$$
\Upsilon(t)=\sum_{d \in \mathbb{N}^{n}} \int_{0}^{t} \xi^{\prime}(r) b_{d} \varphi_{d}\left(e^{i \phi} \cdot \mathrm{d} \beta_{d}\right)
$$

Since $|u| \leq \xi+\frac{1}{2}$, then to estimate $|u|$ it suffices to bound $\xi$. To do that we compare it with a real solution of the stochastic heat equation

$$
\dot{v}-\Delta v=\dot{\Upsilon}, \quad v(0)=v_{0},
$$

where $v_{0}:=\left|\xi_{0}\right|$. We have that $v=v_{1}+v_{2}$, where $v_{1}$ is a solution of (2.5) with $\Upsilon:=0$, and $v_{2}$ is a solution of (2.5) with $v_{0}:=0$. By the maximum principle

$$
\sup _{t \geq 0}\left|v_{1}(t)\right|_{\infty} \leq\left|v_{0}\right|_{\infty} \leq\left|u_{0}\right|_{\infty} .
$$


To estimate $v_{2}$, we use the following lemma established in Appendix to [16] (that proof is reproduced in Appendix below); see [10,11,19] for more general results.

Lemma 2.4 Let $v_{2}$ be a solution of (2.5) with $\dot{\Upsilon}=\sum_{d} b_{d} f^{d}(t, x) \dot{\beta}_{d}(t)$ and $v_{0}=0$, where progressively measurable functions $f^{d}(t, x)$ and real numbers $b_{d}$ are such that $\left|f^{d}(t, x)\right| \leq L$ for each $d$ and $t$ almost surely. Then a.s. $v_{2}$ belongs to $C\left(\mathbb{R}_{+}, C_{0}(K)\right)$, and for any $t \geq 0$ and $p \geq 1$ we have

$$
\mathbb{E} \sup _{s \in[t, t+T]}\left|v_{2}(s)\right|_{\infty}^{2 p} \leq C^{*}(L, T, p) .
$$

Moreover,

$$
\mathbb{E}\left\|\left.v_{2}\right|_{[t, t+1] \times K}\right\|_{C^{\theta / 2, \theta}}^{p} \leq C(p, \theta)
$$

for any $0<\theta<1$, where $\|\cdot\|_{C^{\theta / 2, \theta}}$ is the norm in the Hölder space of functions on $[t, t+1] \times K$.

It is crucial for this work that the constant $C^{*}(L, T, p)$ in (2.7) may be specified:

Lemma 2.5 The constant $C^{*}(L, T, p)$ in Lemma 2.7 may be chosen equal to $\left(C(T) L B_{*}\right)^{2 p} p^{p}$.

This assertion is proved in Appendix, where we follow carefully the constants in the proof of Lemma 2.4, given in [16].

Using the definition of $\xi$ we see that the noise $\Upsilon$ defined by (2.4) verifies the conditions of Lemma 2.6 since the eigen-functions $\varphi_{d}$ satisfy $\left|\varphi_{d}(x)\right| \leq(2 / \pi)^{\frac{n}{2}}$ for all $x \in K$.

Let us denote

$$
h(t, x)=\xi(r(t, x))-v(t, x) .
$$

Since a.s. $u(t, x)$ is uniformly continuous on sets $[0, T] \times K, 0<T<\infty$, then a.s. we can find an open domain $Q=Q^{\omega} \subset[0, \infty) \times K$ with a piecewise smooth boundary $\partial Q$ such that

$$
r \geq \frac{1}{2} \quad \text { in } Q, \quad r \leq \frac{3}{4} \quad \text { outside } Q
$$

Then $h(t, x)$ is a solution of the following problem in $Q$

$$
\begin{aligned}
\dot{h}-\Delta h & =\frac{1}{2 r} \sum_{d \in \mathbb{N}^{n}} b_{d}^{2}\left|\varphi_{d}\right|^{2}-\left(r|\nabla \phi|^{2}+\frac{1}{2 r} \sum_{d \in \mathbb{N}^{n}} b_{d}^{2}\left(e^{i \phi} \cdot \varphi_{d}\right)^{2}\right)=: g(t, x), \\
\left.h\right|_{\partial_{+} Q} & =\left.(r-v)\right|_{\partial_{+} Q}=: m
\end{aligned}
$$

where $\partial_{+} Q$ stands for the parabolic boundary, i.e., the part of the boundary of $Q$ where the external normal makes with the time-axis an angle $\geq \pi / 2$. Note that $m(0, x)=0$. 
We write $h=h_{1}+h_{2}$, where $h_{1}$ is a solution of (2.8), (2.9) with $g=0$ and $h_{2}$ is a solution of (2.8), (2.9) with $m=0$. Since each $\left|\varphi_{d}(x)\right|$ is bounded by $(2 \pi)^{n / 2}$ and $r \geq \frac{1}{2}$ in $Q$, then $g(t, x) \leq(2 / \pi)^{n} B_{0}$ everywhere in $Q$. Now applying the maximum principle (see [17]), we obtain the inequality

$$
\sup _{t \geq 0}\left|h_{2}(t)\right|_{\infty} \leq C B_{0}
$$

(cf. Lemma 6 in [16]). Therefore

$$
|u(t)|_{\infty} \leq \frac{1}{2}+|\xi(r(t))|_{\infty} \leq \frac{1}{2}+C B_{0}+\left|v_{1}(t)\right|_{\infty}+\left|v_{2}(t)\right|_{\infty}+\left|h_{1}(t)\right|_{\infty}
$$

To estimate $h_{1}$ we note that

$$
h_{1}(s, x)=\int_{\partial_{+} Q} m(\xi) G(s, x, \mathrm{~d} \xi)
$$

where $G(s, x, \mathrm{~d} \xi)$ is the Green function ${ }^{3}$ for the problem (2.8), (2.9) with $g=0$, which for any $(s, x) \in Q$ is a probability measure in $Q$, supported by $\partial_{+} Q$. Here we need the following estimate for $G$, proved in [16], Lemma 7, where

$$
Q_{[a, b]}:=Q \cap([a, b] \times K)
$$

Lemma 2.6 Let $0 \leq s \leq t$. Then for any $x \in K$ we have $G\left(t, x, Q_{[0, t-s]}\right)=$ $G\left(t, x, Q_{[0, t-s]} \cap \partial_{+} Q\right) \leq 2^{\frac{n}{2}} e^{-\frac{n \pi^{2}}{4} s}$.

Since $\left.r\right|_{\partial_{+} Q} \leq \frac{3}{4}$, we have

$$
\left|h_{1}(t, x)\right| \leq \frac{3}{4}+\int_{\partial_{+} Q}\left|v_{1}(\xi)\right| G(t, x, \mathrm{~d} \xi)+\int_{\partial_{+} Q}\left|v_{2}(\xi)\right| G(t, x, \mathrm{~d} \xi)
$$

Estimate (2.6) implies

$$
\int_{\partial_{+} Q}\left|v_{1}(\xi)\right| G(t, x, \mathrm{~d} \xi) \leq\left|u_{0}\right|_{\infty}
$$

Let us take a positive constant $T$ and cover the segment $[0, t]$ by segments $I_{1}, \ldots, I_{j_{T}}$, where

$$
j_{T}=\left[\frac{t}{T}\right]+1, \quad I_{j}=[t-T j, t-T j+T]
$$

\footnotetext{
3 It depends on $\omega$, as well as the set $Q$. All estimates below are uniform in $\omega$.
} 
To bound the last integral in (2.11), we apply Lemma 2.6 as follows:

$$
\begin{aligned}
\int_{\partial_{+} Q}\left|v_{2}(\xi)\right| G(t, x, \mathrm{~d} \xi) & \leq \sum_{j=1}^{j_{T}} \int_{Q_{I_{j}}}\left|v_{2}(\xi)\right| G(t, x, \mathrm{~d} \xi) \\
& \leq 2^{\frac{n}{2}} \sum_{j=1}^{j_{T}} e^{-\frac{n \pi^{2}}{4}(j-1) T} \sup _{\tau \in I_{j}}\left|v_{2}(\tau)\right|_{\infty}
\end{aligned}
$$

where $v_{2}(\tau)$ is extended by zero outside $[0, t]$. Denoting

$$
\zeta_{j}=\sup _{\tau \in I_{j}}\left|v_{2}(\tau)\right|_{\infty}, \quad Y=\sum_{j=1}^{j_{T}} e^{-2 j T} \zeta_{j}
$$

and using that $n \pi^{2} / 4>2$ we get

$$
\int_{\partial_{+} Q}\left|v_{2}(\xi)\right| G(t, x, \mathrm{~d} \xi) \leq C Y .
$$

So by (2.12) $\left|h_{1}(t)\right|_{\infty} \leq \frac{3}{4}+\left|u_{0}\right|_{\infty}+C Y$. As $\left|v_{2}(t, x)\right| \leq \zeta_{1} \leq C Y$, then using (2.10) and (2.6) we get for any $u_{0} \in H^{m}$ and any $t \geq 0$ that the solution $u(t, x)$ a.s. satisfies

$$
|u(t, x)| \leq 2\left|u_{0}\right|_{\infty}+C B_{0}+2+C Y
$$

Let us show that there are positive constants $c$ and $C$, not depending on $t$ and $u_{0}$, such that

$$
\mathbb{E}|u(t)|_{\infty}^{2} \leq C e^{-c t}\left|u_{0}\right|_{\infty}^{2}+C \text { for all } t \geq 0
$$

Indeed, since $v_{1}$ is a solution of the free heat equation, then

$$
\left|v_{1}(t)\right|_{\infty} \leq C e^{-c_{1} t}\left|u_{0}\right|_{\infty} \text { for } t \geq 0
$$

This relation, Lemma 2.6 and (2.6) imply that

$$
\begin{aligned}
\int_{\partial_{+} Q}\left|v_{1}(\xi)\right| G(t, x, \mathrm{~d} \xi) & \leq \int_{\partial_{+} Q_{\left[0, \frac{t}{2}\right]}}\left|v_{1}(\xi)\right| G(t, x, \mathrm{~d} \xi)+\int_{\partial_{+} Q_{\left[\frac{t}{2}, t\right]}}\left|v_{1}(\xi)\right| G(t, x, \mathrm{~d} \xi) \\
& \leq \sup _{s \geq 0}\left|v_{1}(s)\right|_{\infty} G\left(t, x, Q_{\left[0, \frac{t}{2}\right]}\right)+\sup _{s \geq \frac{t}{2}}\left|v_{1}(s)\right|_{\infty} \\
& \leq\left|u_{0}\right|_{\infty} 2^{\frac{n}{2}} e^{-\frac{n \pi^{2}}{4} \frac{t}{2}}+C e^{-c_{1} t}\left|u_{0}\right|_{\infty} \leq C e^{-c t}\left|u_{0}\right|_{\infty} .
\end{aligned}
$$


By Lemmas 2.4 and 2.6

$$
\mathbb{E}\left|\int_{\partial_{+} Q} v_{2}(\xi) G(t, x, \mathrm{~d} \xi)\right|^{2} \leq C
$$

for any $t \geq 0$. Combining this with (2.10), (2.11), (2.16) and (2.17), we arrive at (2.15).

Estimates (2.14) and (2.15) are used in the next section to get bounds for exponential moments of $|u|_{\infty}$.

\subsection{Exponential moments of $|u(t)|_{\infty}$}

In this section, we strengthen bounds on polynomial moments of the random variables $\sup _{s \in[t, t+1]}|u(s)|_{\infty}^{2}$, obtained in Theorem 2.2, to bounds on their exponential moments. As a consequence we prove that integrals $\int_{0}^{T}|u(s)|_{\infty}^{2} d s$ have linear growth as functions of $T$ and derive exponential estimates which characterise this growth. These estimates are crucially used in Sects. 3-4 to prove that Eq. (1.2) defines a mixing Markov process.

Theorem 2.7 Under the assumptions of Theorem 2.2, for any $u_{0} \in H^{m}$, any $t \geq 0$ and $T \geq 1$ the solution $u(t, x)$ satisfies the following estimates:

(i) There are constants $c_{*}(T)>0$ and $C(T)>0$, such that for any $c \in\left(0, c_{*}(T)\right]$ we have

$$
\mathbb{E} \exp \left(c \sup _{s \in[t, t+T]}|u(s)|_{\infty}^{2}\right) \leq C(T) \exp \left(5 c\left|u_{0}\right|_{\infty}^{2}\right)
$$

(ii) There are positive constants $\lambda_{0}, C$ and $c_{2}$ such that

$$
\mathbb{E} \exp \left(\lambda \int_{0}^{t}|u(s)|_{\infty}^{2} d s\right) \leq C \exp \left(c_{1}\left|u_{0}\right|_{\infty}^{2}+c_{2} t\right)
$$

for each $\lambda \leq \lambda_{0}$, where $c_{1}=$ Const $\cdot \lambda$.

Proof Step 1 (proof of (i)). Due to (2.14), to prove (2.18) we have to estimate exponential moments of $Y^{2}$. First let us show that for a suitable $C_{2}(T)>0$ we have

$$
\mathbb{E} \exp \left(c \sup _{s \in[t, t+T]}\left|v_{2}(s)\right|_{\infty}^{2}\right) \leq \frac{1}{1-c C_{2}(T)} \text { for any } t \geq 0 \text { and } c<\frac{1}{C_{2}(T)}
$$


Indeed, using (2.7) and Lemma 2.5 we get

$$
\begin{aligned}
\mathbb{E} \exp \left(c \sup _{[t, t+T]}\left|v_{2}(s)\right|_{\infty}^{2}\right) & =\mathbb{E} \sum_{p=0}^{\infty} \frac{c^{p} \sup _{[t, t+T]}\left|v_{2}(s)\right|_{\infty}^{2 p}}{p !} \leq \sum_{p=0}^{\infty} \frac{c^{p}\left(C(T) B_{*}\right)^{2 p} p^{p}}{p !} \\
& \leq \sum_{p=0}^{\infty}\left(c e\left(C(T) B_{*}\right)^{2}\right)^{p} \leq \frac{1}{1-c e\left(C(T) B_{*}\right)^{2}}
\end{aligned}
$$

since $p ! \geq(p / e)^{p}$. Thus we get (2.20) with $C_{2}:=e\left(C(T) B_{*}\right)^{2}$. In particular,

$$
\mathbb{E} e^{c^{\prime} \zeta_{j}^{2}} \leq\left(1-c^{\prime} C_{2}(T)\right)^{-1} \quad \forall c^{\prime} \leq c
$$

Next we note that since

$$
Y^{2} \leq C^{2}\left(\sum_{j=1}^{j_{T}} e^{-j}\left(e^{-j} \zeta_{j}\right)\right)^{2} \leq 2 C^{2} \sum_{j=1}^{j_{T}} e^{-2 j} \zeta_{j}^{2}
$$

by Cauchy-Schwarz (we use that $T \geq 1$ ), then

$$
\mathbb{E} e^{c^{\prime} Y^{2}} \leq \mathbb{E} \prod_{j=0}^{j_{T}} e^{2 c^{\prime} C^{2} 5^{-j} \zeta_{j}^{2}}
$$

as $e^{2}>5$. Denote $p_{j}=\alpha 2^{j}, j \geq 0$. Choosing $\alpha \in(1,2)$ in a such a way that $\sum_{j=0}^{j_{T}}\left(1 / p_{j}\right)=1$, using the Hölder inequality with these $p_{j}$ 's and (2.21), we find that

$$
\begin{aligned}
\mathbb{E} e^{c^{\prime} Y^{2}} & \leq \prod_{j=0}^{j_{T}}\left(\mathbb{E} e^{2 p_{j} c^{\prime} C^{2} 5^{-j} \zeta_{j}^{2}}\right)^{\frac{1}{p_{j}}} \leq \prod_{j=0}^{j_{T}}\left(\mathbb{E} e^{2 c^{\prime} C^{2} \zeta_{j}^{2}}\right)^{\frac{1}{p_{j}}} \\
& \leq \prod_{j=0}^{j_{T}}\left(1-c^{\prime} C_{3}(T)\right)^{-\frac{1}{p_{j}}}=\exp \left(-\sum_{j=0}^{j_{T}} p_{j}^{-1} \ln \left(1-c^{\prime} C_{3}\right)\right) \leq e^{c^{\prime} C_{4}(T)}
\end{aligned}
$$

if $2 c^{\prime} C^{2} \leq c$ and $c^{\prime} \leq\left(2 C_{3}(T)\right)^{-1}$. In view of (2.14), this implies (2.18).

Step 2. Now we show that for any $A \geq 1$ there is a time $T(A)$ such that for $T \geq T(A)$ we have

$$
\mathbb{E} \exp \left(c\left(\sup _{s \in[0, T]}|u(s)|_{\infty}^{2}+A|u(T)|_{\infty}^{2}\right)\right) \leq \tilde{C} \exp \left(6 c\left|u_{0}\right|_{\infty}^{2}\right)
$$

for any $c \in(0, \tilde{c}]$, where $\tilde{C}$ and $\tilde{c}$ depend on $A$ and $T$. 
Indeed, due to (2.10) and (2.16),

$$
|u(T)|_{\infty} \leq 2+C e^{-c T}\left|u_{0}\right|_{\infty}+C B_{0}+\left|v_{2}(T)\right|_{\infty}+\left|h_{1}(T)\right|_{\infty}
$$

By (2.11), (2.17) and (2.13), $\left|h_{1}(T)\right|_{\infty} \leq \frac{3}{4}+C Y+C e^{-c^{\prime} T}\left|u_{0}\right|_{\infty}$. Therefore choosing a suitable $T=T(A)$ we achieve that

$$
c A|u(T)|_{\infty}^{2} \leq c\left(C_{1} A+C_{2} A Y^{2}+\left|u_{0}\right|_{\infty}^{2}\right)+2 c A\left|v_{2}(T)\right|_{\infty}^{2}
$$

Using Hölder's inequality we see that the cube of the term in the 1.h.s. of (2.23) is bounded by

$$
C(A) e^{3 c\left|u_{0}\right|_{\infty}^{2}} \mathbb{E} e^{3 c C_{2} A Y^{2}} \mathbb{E} e^{6 c A\left|v_{2}(T)\right|_{\infty}^{2}} \mathbb{E} e^{3 c \sup _{s \in[0, T]}|u(s)|_{\infty}^{2}}
$$

Taking $c \leq c(A)$ and using (2.22), (2.20) and (2.18) we estimate the product by $C(A, T) e^{3 c\left|u_{0}\right|_{\infty}^{2}} e^{15 c\left|u_{0}\right|_{\infty}^{2}}$. This implies (2.23).

Step 3. (proof of (ii)). Let $T_{0} \geq 1$ be such that (2.23) holds with $A=6$. Let $c>0$ and $C>0$ be the constants in (2.18), corresponding to $T=T_{0}$, and let $\lambda \leq c / T_{0}$. It suffices to prove (2.19) for $t=T_{0} k, k \in \mathbb{N}$, since this result implies (2.19) with any $t \geq 0$ if we modify the constant $C$. By the Markov property,

$$
\begin{aligned}
X_{\lambda}:=\mathbb{E}_{u_{0}} \exp \left(\lambda \int_{0}^{T_{0} k}|u(s)|_{\infty}^{2} \mathrm{~d} s\right)= & \mathbb{E}_{u_{0}}\left(\exp \left(\lambda \int_{0}^{T_{0}(k-1)}|u(s)|_{\infty}^{2} \mathrm{~d} s\right)\right. \\
& \left.\times \mathbb{E}_{u\left(T_{0}(k-1)\right)} \exp \left(\lambda \int_{0}^{T_{0}}|u(s)|_{\infty}^{2} \mathrm{~d} s\right)\right),
\end{aligned}
$$

and by (2.18)

$$
\mathbb{E}_{u\left(T_{0}(k-1)\right)} \exp \left(\lambda \int_{0}^{T_{0}}|u(s)|_{\infty}^{2} \mathrm{~d} s\right) \leq C \exp \left(5 \lambda T_{0}\left|u\left(T_{0}(k-1)\right)\right|_{\infty}^{2}\right)
$$

Combining these two relations we get

$$
X_{\lambda} \leq C \mathbb{E}_{u_{0}} \exp \left(\lambda \int_{0}^{T_{0}(k-1)}|u(s)|_{\infty}^{2} \mathrm{~d} s+6 T_{0} \mid u\left(\left.T_{0}(k-1)\right|_{\infty} ^{2}\right)\right.
$$


Applying again the Markov property and using (2.23) with $A=6$ and $c=\lambda T_{0}$ we obtain

$$
\begin{aligned}
X_{\lambda} \leq & C \mathbb{E}_{u_{0}}\left(\exp \left(\lambda \int_{0}^{T_{0}(k-2)}|u(s)|_{\infty}^{2} \mathrm{~d} s\right)\right. \\
& \left.\times \mathbb{E}_{u\left(\left(T_{0}(k-2)\right)\right.} \exp \left(\lambda T_{0}\left(\sup _{0 \leq s \leq T_{0}}|u(s)|_{\infty}^{2}+6\left|u\left(T_{0}\right)\right|_{\infty}^{2}\right)\right)\right) \\
\leq & C^{2} \mathbb{E}_{u_{0}} \exp \left(\lambda \int_{0}^{T_{0}(k-2)}|u(s)|_{\infty}^{2} \mathrm{~d} s+6 \lambda T_{0}\left|u\left(T_{0}(k-2)\right)\right|_{\infty}^{2}\right)
\end{aligned}
$$

Iteration gives

$$
X_{\lambda} \leq C^{m} \mathbb{E}_{u_{0}} \exp \left(\lambda \int_{0}^{T_{0}(k-m)}|u(s)|_{\infty}^{2} \mathrm{~d} s+6 \lambda T_{0}\left|u\left(T_{0}(k-m)\right)\right|_{\infty}^{2}\right)
$$

for any $m \leq k$. When $m=k$, this relation proves (2.19) with $t=k T_{0}, C=1, c_{1}=$ $6 \lambda T_{0}$ and a suitable $c_{2}$.

In the lemma below by $c_{1}, c_{2}$ and $\lambda_{0}$ we denote the constants from Theorem 2.7(ii).

Lemma 2.8 For any $u_{0} \in H^{m}$ the solution $u(t, x)$ satisfies the following estimate for any $\rho \geq 0$

$$
\mathbb{P}\left\{\sup _{t \geq 0}\left(\int_{0}^{t}|u(s)|_{\infty}^{2} d s-K t\right) \geq \rho\right\} \leq C^{\prime} \exp \left(c_{1}|u|_{\infty}^{2}-\lambda \rho\right)
$$

where $C^{\prime}$ is an absolute constant, $K=\lambda^{-1}\left(c_{2}+1\right)$ and $\lambda$ is a suitable constant from $\left(0, \lambda_{0}\right]$.

Proof For any real number $t$ denote $\lceil t\rceil=\min \{n \in \mathbb{Z}: n \geq t\}$. Then

$$
\left\{\left(\int_{0}^{t}|u|_{\infty}^{2} d s-K t\right) \geq \rho\right\} \subset\left\{\left(\int_{0}^{\lceil t\rceil}|u|_{\infty}^{2} d s-K\lceil t\rceil\right) \geq \rho-K\right\}
$$

So it suffices to prove (2.24) for integer $t$ since then the required inequality follows with a modified constant $C^{\prime}$. Accordingly below we replace $\sup _{t \geq 0}$ by $\sup _{n \in \mathbb{N}}$. By the Chebyshev inequality and estimate (2.19) we have 


$$
\begin{aligned}
\mathbb{P}\left\{\sup _{n \in \mathbb{N}}\left(\int_{0}^{n}|u(s)|_{\infty}^{2} \mathrm{~d} s-K n\right) \geq \rho\right\} & \leq \sum_{n \in \mathbb{N}} \mathbb{P}\left\{\int_{0}^{n}|u(s)|_{\infty}^{2} \mathrm{~d} s \geq \rho+K n\right\} \\
& \leq \sum_{n \in \mathbb{N}} \exp (-\lambda(\rho+K n)) C \exp \left(c_{1}\left|u_{0}\right|_{\infty}^{2}+c_{2} n\right) \\
& \leq C \exp \left(-\lambda \rho+c_{1}\left|u_{0}\right|_{\infty}^{2}\right) \sum_{n \in \mathbb{N}} \exp (-n) \\
& =C^{\prime} \exp \left(c_{1}\left|u_{0}\right|_{\infty}^{2}-\lambda \rho\right)
\end{aligned}
$$

since $\lambda K-c_{2}=1$. This proves (2.24).

\section{Markov process in $C_{0}(K)$}

The goal of this section is to construct a family of Markov processes, associated with Eq. (1.2) in the space $C_{0}(K)$. To this end we first prove a well-posedness result in that space.

\subsection{Existence and uniqueness of solutions}

Let $u_{0} \in C_{0}(K)$. Denote by $\Pi_{m}: H \rightarrow \mathbb{C}^{m}$ the usual Galerkin projection and set $\eta^{m}:=\Pi_{m} \eta=: \frac{\partial}{\partial t} \zeta^{m}$. Let $u_{0}^{m} \in C^{\infty}$ be such that $\left|u_{0}^{m}-u_{0}\right|_{\infty} \rightarrow 0$ as $m \rightarrow \infty$ and $\left|u_{0}^{m}\right|_{\infty} \leq\left|u_{0}\right|_{\infty}+1$. Let $u^{m}$ be a solution of (1.2), (1.6) with regular right-hand side $\eta=\eta^{m}$ and regular initial condition $u_{0}=u_{0}^{m}$, existing by Theorem 2.2.

Fix any $T>0$. For $\alpha \in(0,1)$ and a Banach space $X$, let $C^{\alpha}([0, T], X)$ be the space of all $u \in C([0, T], X)$ such that

$$
\|u\|_{C^{\alpha}([0, T], X)}:=\|u\|_{C([0, T], X)}+\sup _{0 \leq t_{1}<t_{2} \leq T} \frac{\left|u\left(t_{2}\right)-u\left(t_{1}\right)\right|}{\left|t_{2}-t_{1}\right|^{\alpha}}<\infty .
$$

Let us define the spaces

$$
\begin{aligned}
& \mathcal{U}:=L^{2}\left([0, T], H^{1}\right) \cap C^{\alpha}\left([0, T], H^{-1}\right), \\
& \mathcal{V}:=L^{2}\left([0, T], H^{1-\varepsilon}\right) \cap C\left([0, T], H^{-2}\right),
\end{aligned}
$$

where $\alpha \in\left(0, \frac{1}{2}\right)$ and $\varepsilon>0$. Then

$$
\text { space } \mathcal{U} \text { is compactly embedded into } \mathcal{V} \text {. }
$$

Indeed, by Theorem 5.2 in [18], $\mathcal{U} \Subset L^{2}\left([0, T], H^{1-\varepsilon}\right) .{ }^{4}$ On the other hand, $C^{\alpha}\left([0, T], H^{-1}\right) \Subset C\left([0, T], H^{-2}\right)$, by the Arzelà-Ascoli theorem.

\footnotetext{
$\overline{{ }^{4} \text { One should note that if } u(t)}=\sum u_{d}(t) \varphi_{d} \in C^{\alpha}\left([0, T], H^{-1}\right)$ and $\|u\|_{C^{\alpha}\left([0, T], H^{-1}\right)} \leq 1$, then $u$ belongs to the space denoted in [18] by $\mathscr{H}^{\alpha}\left(0, T ; H^{-1}, H^{-N}\right)=: \mathscr{H}$ and $\|u\|_{\mathscr{H}} \leq C(\alpha, N)$ for a suitable $N$, since for each $d$ we have $\left\|u_{d}\right\|_{H^{\alpha}([0, T])} \leq C\left\|u_{d}\right\|_{C^{\alpha}([0, T])} \leq C_{1}|d|$ (for $\alpha=0$ or $\alpha=1$ this is obvious, and for $0<\alpha<1$ this follows by interpolation).
} 
Lemma 3.1 For $m \geq 1$ let $M_{m}$ be the law of the solution $\left\{u^{m}\right\}$, constructed above. Then

(i) The sequence $\left\{M_{m}\right\}$ is tight in $\mathcal{V}$.

(ii) Any limiting measure $M$ of $M_{m}$ is the law of a weak solution $\tilde{u}(t), 0 \leq t \leq T$, of (1.2), (1.6). This solution satisfies (2.1) for $0 \leq t \leq T-1$ and (2.2), (2.18), (2.24) for $0 \leq t \leq T$.

(iii) If $1 \leq t \leq T-1$, then for any $0<\theta<1$ and any $q \geq 1$ we have

$$
\mathbb{E}\left\|\left.\tilde{u}\right|_{[t, t+1] \times K}\right\|_{C^{\theta / 2, \theta}}^{q} \leq C\left(q, \theta,\left|u_{0}\right|_{\infty}\right) .
$$

Proof The process $u^{m}$ satisfies the following equation with probability 1

$$
u^{m}(t)=u_{0}^{m}+\int_{0}^{t}\left(\Delta u^{m}-i\left|u^{m}\right|^{2} u^{m}\right) \mathrm{d} s+\zeta^{m}=: V^{m}+\zeta^{m} .
$$

Using (2.1) and (2.2), we get

$$
\mathbb{E}\left\|V^{m}\right\|_{W^{1,2}\left([0, T], H^{-1}\right)}^{2} \leq C .
$$

It is well known that Brownian motion $\beta_{d}$ satisfies $^{5}$

$$
\mathbb{E}\left|\beta_{d}\right|_{C^{\alpha}([0, T])}^{2} \leq C_{\alpha},
$$

(e.g., see [25], Chapter X, §2). Since for any $0 \leq t_{1}<t_{2} \leq T$ we have

$$
\left|t_{2}-t_{1}\right|^{-2 \alpha}\left\|\zeta^{m}\left(t_{2}\right)-\zeta^{m}\left(t_{1}\right)\right\|_{-1}^{2} \leq \sum_{d}|d|^{-2} b_{d}^{2}\left|\beta_{d}\right|_{C^{\alpha}([0, T])}^{2},
$$

then for any $m \geq 1$ we get

$$
\mathbb{E}\left\|\zeta^{m}\right\|_{C^{\alpha}\left([0, T], H^{-1}\right)}^{2} \leq C_{\alpha} B_{-1}^{2} \leq C_{\alpha} B_{*}^{2}
$$

Combining (3.3) and (3.4), we obtain

$$
\mathbb{E}\left\|u^{m}\right\|_{C^{\alpha}\left([0, T], H^{-1}\right)}^{2} \leq 2 \mathbb{E}\left\|V^{m}\right\|_{C^{\alpha}\left([0, T], H^{-1}\right)}^{2}+2 \mathbb{E}\left\|\zeta^{m}\right\|_{C^{\alpha}\left([0, T], H^{-1}\right)}^{2} \leq C .
$$

Jointly with (2.2) this estimate implies that $\mathbb{E}\left\|u^{m}\right\|_{\mathcal{U}}^{2} \leq C_{1}$ for each $m$ with a suitable $C_{1}$. Now (i) holds by (3.1) and the Prokhorov theorem.

Let us prove (ii). Suppose that $M_{m}$ converges weakly to $M$ in $\mathcal{V}$. By Skorohod's embedding theorem, there is a probability space $(\tilde{\Omega}, \tilde{\mathcal{F}}, \tilde{\mathbb{P}})$ and $\mathcal{V}$-valued random

\footnotetext{
5 By the Kolmogorov-Chentsov theorem, $\beta_{d} \in C^{\alpha}([0, T])$ a.s. So $|\cdot|_{C^{\alpha}([0, T])}$ is a measurable seminorm for the Gaussian process $\beta_{d}$, and by the Fernique theorem $\mathbb{E} \exp \left(\sigma\left|\beta_{d}\right|_{C^{\alpha}([0, T])}^{2}\right)<\infty$ for some positive $\sigma$; see [2]. This also implies the estimate.
} 
variables $\tilde{u}^{m}$ and $\tilde{u}$, defined on it, such that each $\tilde{u}^{m}$ is distributed as $M_{m}, \tilde{u}$ is distributed as $M$ and $\mathbb{P}$-a.s. we have $\tilde{u}^{m} \rightarrow \tilde{u}$ in $\mathcal{V}$.

Since $\mathcal{V} \subset L_{2}([0, T] \times K)=: L_{2}$, then $\tilde{u}^{m} \rightarrow \tilde{u}$ in $L_{2}$, a.s. For any $R \in(0, \infty]$ and $p, q \in[1, \infty)$ consider the functional $f_{R}^{p}$,

$$
f_{R}^{p}(u)=\left.\left.|| u\right|^{q} \wedge R\right|_{L^{p}([t, t+1] \times K)} \leq \pi^{\frac{n}{p}}|u|_{L^{\infty}([t, t+1] \times K)}^{q} .
$$

Since for $p, R<\infty$ it is continuous in $L_{2}$, then by (2.1) we have

$$
\mathbb{E}\left(f_{R}^{p}(\tilde{u})\right)=\lim _{m \rightarrow \infty}\left(f_{R}^{p}\left(\tilde{u}^{m}\right)\right) \leq \pi^{\frac{n}{p}} C_{q} \text { for } p, R<\infty .
$$

As for each $v(t, x) \in L^{\infty}([t, t+1] \times K)$ the function $[1, \infty] \ni p \mapsto|v|_{L^{p}([t, t+1] \times K)} \in$ $[0, \infty]$ is continuous and non-decreasing, then sending $p$ and $R$ to $\infty$ and using the monotone convergence theorem, we get $\mathbb{E} \sup _{s \in[t, t+1]}|\tilde{u}(s)|_{\infty}^{q} \leq C_{q}$. I.e., $\tilde{u}$ satisfies (2.1).

By (2.2) for each $m$ and $N$ we have

$$
\mathbb{E}\left\|\Pi_{N} \tilde{u}^{m}(t)\right\|^{2}+2 \mathbb{E} \int_{0}^{t}\left\|\Pi_{N} \tilde{u}^{m}(\tau)\right\|_{1}^{2} \mathrm{~d} \tau \leq\left\|u_{0}^{m}\right\|^{2}+B_{0} t .
$$

Passing to the limit as $m \rightarrow \infty$ and then $N \rightarrow \infty$ and using the monotone convergence theorem, we obtain that $\tilde{u}$ satisfies (2.2), where the equality sign is replace by $\leq$. We will call this estimate $(2.2)_{\leq}$.

By the same reason (cf. Lemma 1.2.17 in [14]) the process $\tilde{u}(t)$ satisfies (2.18) and (2.24).

Since $\tilde{u}^{m}$ is a weak solution of the equation, then

$$
\tilde{u}^{m}(t)-u_{0}^{m}-\int_{0}^{t}\left(\Delta \tilde{u}^{m}-i\left|\tilde{u}^{m}\right|^{2} \tilde{u}^{m}\right) \mathrm{d} s=\tilde{\zeta}^{m},
$$

where $\tilde{\zeta}^{m}$ is distributed as the process $\zeta$. Using the Cauchy-Schwarz inequality and (2.1), we get

$$
\begin{aligned}
& \mathbb{E} \int_{0}^{T}\left\|\left|\tilde{u}^{m}\right|^{2} \tilde{u}^{m}-|\tilde{u}|^{2} \tilde{u}\right\| \mathrm{d} s \leq C \mathbb{E} \int_{0}^{T}\left\|\left(\tilde{u}^{m}-\tilde{u}\right)\left(\left|\tilde{u}^{m}\right|^{2}+|\tilde{u}|^{2}\right)\right\| \mathrm{d} s \\
& \quad \leq C \mathbb{E} \sup _{t \in[0, T]}\left(\left|\tilde{u}^{m}(t)\right|_{\infty}^{2}+|\tilde{u}(t)|_{\infty}^{2}\right) \int_{0}^{T}\left\|\tilde{u}^{m}-\tilde{u}\right\| \mathrm{d} s \\
& \quad \leq C \sqrt{T}\left(\mathbb{E} \sup _{t \in[0, T]}\left(\left|\tilde{u}^{m}(t)\right|_{\infty}^{4}+|\tilde{u}(t)|_{\infty}^{4}\right)\right)^{\frac{1}{2}}\left(\mathbb{E} \int_{0}^{T}\left\|\tilde{u}^{m}-\tilde{u}\right\|^{2} \mathrm{~d} s\right)^{\frac{1}{2}}
\end{aligned}
$$




$$
\leq C\left(T,\left|u_{0}\right|_{\infty}\right)\left(\mathbb{E} \int_{0}^{T}\left\|\tilde{u}^{m}-\tilde{u}\right\|^{2} \mathrm{~d} s\right)^{\frac{1}{2}} .
$$

Since the r.h.s. goes to zero when $m \rightarrow \infty$, then for a suitable subsequence $m_{k} \rightarrow \infty$ we have a.s.

$$
\left\|\int_{0}^{t}\left|\tilde{u}^{m_{k}}\right|^{2} \tilde{u}^{m_{k}} \mathrm{~d} s-\int_{0}^{t}|\tilde{u}|^{2} \tilde{u} \mathrm{~d} s\right\|_{C\left([0, T], L^{2}\right)} \rightarrow 0 \text { as } k \rightarrow \infty .
$$

Therefore the 1.h.s. of (3.5) converges to $\left(\tilde{u}(t)-u_{0}-\int_{0}^{t}\left(\Delta \tilde{u}-i|\tilde{u}|^{2} \tilde{u}\right) \mathrm{d} s\right)$ in the space $C\left([0, T], H^{-2}\right)$ over the sequence $\left\{m_{k}\right\}$, a.s. So a.s. there exists a $\operatorname{limit} \lim \tilde{\zeta}^{m_{k}}(\cdot)=$ $\tilde{\zeta}(\cdot)$, and

$$
\tilde{u}(t)-u_{0}-\int_{0}^{t}\left(\Delta \tilde{u}-i|\tilde{u}|^{2} \tilde{u}\right) \mathrm{d} s=\tilde{\zeta}(t)
$$

We immediately get that $\tilde{\zeta}(t)$ is a Wiener process in $H^{-2}$, distributed as the process $\zeta$. Let $\tilde{\mathcal{F}}_{t}, t \geq 0$, be a sigma-algebra, generated by $\{\tilde{u}(s), 0 \leq s \leq t\}$ and the zero-sets of the measure $\tilde{\mathbb{P}}$. From (3.6), $\tilde{\zeta}(t)$ is $\tilde{\mathcal{F}}_{t}$-measurable. So $\tilde{\zeta}(t)$ is a Wiener process on the filtered probability space $\left(\tilde{\Omega}, \tilde{\mathcal{F}},\left\{\tilde{\mathcal{F}}_{t}\right\}, \tilde{\mathbb{P}}\right)$, distributed as $\zeta$.

Since $\tilde{u}(t, x)$ satisfies (3.6), we can write $\tilde{u}=u_{1}+u_{2}+u_{3}$, where $u_{1}$ satisfies (2.5) with $\dot{\Upsilon}=0, v_{0}=u_{0} ; u_{2}$ satisfies (2.5) with $\dot{\Upsilon}=-i|\tilde{u}|^{2} \tilde{u}, v_{0}=0$ and $u_{3}$ satisfies (2.5) with $\Upsilon=\tilde{\zeta}, v_{0}=0$. Now Lemma 2.4 and the parabolic regularity imply that $\tilde{u} \in C\left([0, T] ; C_{0}(K)\right)$, a.s. As $\tilde{u}$ satisfies $(2.2)_{\leq}$, then $\tilde{u} \in \mathcal{H}([0, T])$ a.s. Since clearly $\tilde{u}(0)=u_{0}$ a.s., then $\tilde{u}$ is a weak solution of (1.2), (1.6).

Regarding $\tilde{u}(t)$ as an Ito process in the space $H$, using (2.1) and applying to $\|\tilde{u}(t)\|^{2}$ the Ito formula in the form, given in [14], we see that $\|\tilde{u}(t)\|^{2}$ satisfies the relation, given by the displayed formula above (2.2). Taking the expectation we recover for $\tilde{u}$ the equality (2.2).

It remains to prove (iii). Functions $u_{1}$ and $u_{3}$ meet (3.2) by Lemma 2.4 and the parabolic regularity. Consider $u_{2}$. Since $u_{2}=\tilde{u}-u_{1}-u_{3}$, then $u_{2}$ satisfies (2.1). Consider restriction of $u_{2}$ to the cylinder $[t-1, t+1] \times K$. Since $u_{2}$ satisfies the heat equation, where the r.h.s. and the Cauchy data at $(t-1) \times K$ are bounded functions, then by the parabolic regularity restriction of $u_{2}$ to $[t, t+1] \times K$ also meets (3.2).

The pathwise uniqueness property holds for the constructed solutions:

Lemma 3.2 Let $u(t)$ and $v(t), t \in[0, T]$, be processes in the space $C_{0}(K)$, defined on some probability space, and let $\zeta(t)$ be a Wiener process, defined on the same space and distributed as $\zeta$ in (1.3). Assume that a.s. trajectories of $u$ and $v$ belong to $\mathcal{H}([0, T])$ and satisfy (1.2), (1.6). Then $u(t) \equiv v(t)$ a.s. 
Proof For any $R>0$ let us introduce the stopping time

$$
\tau_{R}=\inf \left\{t \in[0, T]:|u(t)|_{\infty} \vee|v(t)|_{\infty} \geq R\right\}
$$

The difference $w:=u-v$ satisfies

$$
\dot{w}-\Delta w+i\left(|u|^{2} u-|v|^{2} v\right)=0, \quad w(0)=0 .
$$

Taking the scalar product in $H$ of this equation with $w$ when $t \leq \tau_{R}$ and applying the Gronwall inequality, we get that $w(t) \equiv 0$ for $t \leq \tau_{R}$. Since $u, v \in \mathcal{H}([0, T])$, then $\tau_{R} \rightarrow T$, a.s. as $R \rightarrow \infty$. Therefore $w(t) \equiv 0$ for all $t \in[0, T]$, a.s. This completes the proof.

By the Yamada-Watanabe arguments (e.g., see [12]), existence of a weak solution plus pathwise uniqueness implies the existence of a unique strong solution $u(t), 0 \leq$ $t \leq T$. Since $T$ is any positive number, we get

Theorem 3.3 Let $u_{0} \in C_{0}(K)$. Then problem (1.2), (1.6) has a unique strong solution $u(t), t \geq 0$. This solutions satisfies relations (2.1), (2.2), (2.18) and (2.24); for $t \geq 1$ it also satisfies (3.2).

\subsection{Markov process}

Let us denote by $u(t)=u\left(t, u_{0}\right)$ the unique solution of (1.2), corresponding to an initial condition $u_{0} \in C_{0}(K)$. Equation (1.2) defines a family of Markov process in the space $C_{0}(K)$, parametrized by $u_{0}$. For any $u \in C_{0}(K)$ and $\Gamma \in \mathcal{B}\left(C_{0}(K)\right)$, we set $P_{t}(u, \Gamma)=\mathbb{P}\{u(t, u) \in \Gamma\}$. The Markov operators, corresponding to the process $u(t)$, have the form

$$
\mathfrak{P}_{t} f(u)=\int_{C_{0}(K)} P_{t}(u, \mathrm{~d} v) f(v), \quad \mathfrak{P}_{t}^{*} \mu(\Gamma)=\int_{C_{0}(K)} P_{t}(u, \Gamma) \mu(\mathrm{d} u),
$$

where $f \in C_{b}\left(C_{0}(K)\right)$ and $\mu \in \mathcal{P}\left(C_{0}(K)\right)$.

Lemma 3.4 The Markov process associated with (1.2) is Feller.

Proof We need to prove that $\mathfrak{P}_{t} f \in C_{b}\left(C_{0}(K)\right)$ for any $f \in C_{b}\left(C_{0}(K)\right)$ and $t>0$. To this end, let us take any $u_{0}, v_{0} \in C_{0}(K)$, and let $u$ and $v$ be the corresponding solutions of (1.2) given by Theorem 3.3. Let us take any $R>R_{0}:=\left|u_{0}\right|_{\infty} \vee\left|v_{0}\right|_{\infty}$. Let $\tau_{R}$ be the stopping time defined by (3.7), and let $u_{R}(t):=u\left(t \wedge \tau_{R}\right)$ and $v_{R}(t):=v\left(t \wedge \tau_{R}\right)$ be the stopped solutions. Then

$$
\begin{aligned}
\left|\mathfrak{P}_{t} f\left(u_{0}\right)-\mathfrak{P}_{t} f\left(v_{0}\right)\right| \leq & \mathbb{E}\left|f(u)-f\left(u_{R}\right)\right|+\mathbb{E}\left|f(v)-f\left(v_{R}\right)\right| \\
& +\mathbb{E}\left|f\left(u_{R}\right)-f\left(v_{R}\right)\right|=: I_{1}+I_{2}+I_{3}
\end{aligned}
$$


By (2.1) and the Chebyshev inequality, we have

$$
\begin{aligned}
\max \left\{I_{1}, I_{2}\right\} & \leq 2|f|_{\infty} \mathbb{P}\left\{t>\tau_{R}\right\} \leq 2|f|_{\infty} \mathbb{P}\{U(t) \vee V(t)>R\} \\
& \leq \frac{4}{R}|f|_{\infty} \sup _{\left|u_{0}\right|_{\infty} \leq R_{0}} \mathbb{E} U(t) \rightarrow 0 \text { as } R \rightarrow \infty,
\end{aligned}
$$

where $U(t)=\sup _{s \in[0, t]}|u(s)|_{\infty}$ and $V(t)$ is defined similarly. To estimate $I_{3}$, notice that $w=u-v$ is a solution of

$$
\dot{w}-\Delta w+i\left(|u|^{2} u-|v|^{2} v\right)=0, \quad w(0)=u_{0}-v_{0}=: w_{0} .
$$

We rewrite this in the Duhamel form

$$
w=e^{t \Delta} w_{0}-i \int_{0}^{t} e^{(t-s) \Delta}\left(|u|^{2} u-|v|^{2} v\right) \mathrm{d} s .
$$

Since, by the maximum principle, $\left|e^{t \Delta} z\right|_{\infty} \leq|z|_{\infty}$, then

$$
\begin{aligned}
\left|w\left(t \wedge \tau_{R}\right)\right|_{\infty} \leq\left|w_{0}\right|_{\infty} & +\left.\int_{0}^{t \wedge \tau_{R}}|| u\right|^{2} u-\left.|v|^{2} v\right|_{\infty} \mathrm{d} s \leq\left|w_{0}\right|_{\infty} \\
& +3 \int_{0}^{t \wedge \tau_{R}}\left(|u|_{\infty}^{2}+|v|_{\infty}^{2}\right)|w|_{\infty} \mathrm{d} s .
\end{aligned}
$$

By the Gronwall inequality, $I_{3} \leq \mathbb{E}\left|w\left(t \wedge \tau_{R}\right)\right|_{\infty} \leq\left|w_{0}\right|_{\infty} e^{t C_{R}} \rightarrow 0$ as $\left|w_{0}\right|_{\infty} \rightarrow 0$. Therefore the function $\mathfrak{P}_{t} f(u)$ is continuous in $u \in C_{0}(K)$, as stated.

A measure $\mu \in \mathcal{P}\left(C_{0}(K)\right)$ is said to be stationary for Eq. (1.2) if $\mathfrak{P}_{t}^{*} \mu=\mu$ for every $t \geq 0$. The following theorem is proved in the standard way by applying the Bogolyubov-Krylov argument (e.g. see in [14]).

Theorem 3.5 Equation (1.2) has at least one stationary measure $\mu$, satisfying $\int_{H^{1}}\|u\|_{1}^{2} \mu(d u)=\frac{1}{2} B_{0}$ and $\int_{C_{0}(K)} e^{c|u|_{\infty}^{2}} \mu(d u)<\infty$ for any $c<c_{*}$, where $c_{*}>0$ is the constant in assertion (i) of Theorem 2.7 .

\subsection{Estimates for some hitting times}

For any $r, L, R>0$ we introduce the following hitting times for a solution $u(t)$ of (1.2):

$$
\begin{aligned}
\tau_{1, r, L} & :=\inf \left\{t \geq 0:\|u(t)\| \leq r,|u(t)|_{\infty} \leq L\right\}, \\
\tau_{2, R} & :=\inf \left\{t \geq 0:|u(t)|_{\infty} \leq R\right\} .
\end{aligned}
$$


Lemma 3.6 There is a constant $L>0$ such that for any $r>0$ we have

$$
\mathbb{E} e^{\gamma \tau_{1, r, L}} \leq C\left(1+|u(0)|_{\infty}^{2}\right)
$$

where $\gamma$ and $C$ are suitable positive constants, depending on $r$ and $L$.

It is well known that inequality (3.8) follows from the two statements below (see Proposition 2.3 in [22] or Section 3.3.2 in [14]).

Lemma 3.7 There are positive constants $\delta, R$ and $C$ such that

$$
\mathbb{E} e^{\delta \tau_{2, R}} \leq C\left(1+|u(0)|_{\infty}^{2}\right)
$$

Lemma 3.8 For any $R>0$ and $r>0$ there is a non-random time $T>0$ and positive constants $p$ and $L$ such that

$$
\mathbb{P}\left\{u\left(T, u_{0}\right) \in B_{H}(r) \cap B_{C_{0}(K)}(L)\right\} \geq p \text { for any } u_{0} \in B_{C_{0}(K)}(R) .
$$

Proof of Lemma 3.7 Let us consider the function $F(u)=\max \left(|u|_{\infty}^{2}, 1\right)$. We claim that this is a Lyapunov function for Eq. (1.2). That is,

$$
\mathbb{E} F(u(T, u)) \leq a F(u) \quad \text { for }|u|_{\infty} \geq R^{\prime},
$$

for suitable $a \in(0,1), T>0$ and $R^{\prime}>0$. Indeed, let $|u|_{\infty} \geq R^{\prime}$ and $T>1$. Since $F(u) \leq 1+|u|_{\infty}^{2}$, then

$$
\mathbb{E} F(u(T, u)) \leq 1+\mathbb{E}|u(T, u)|_{\infty}^{2} \leq 1+C e^{-c T}|u|_{\infty}^{2}+C,
$$

where we used (2.15).

This implies (3.10). Since due to (2.15) for $|u|_{\infty}<R^{\prime}$ and any $T>1$ we have $\mathbb{E} F(u(T, u)) \leq C^{\prime}$ then (3.9) follows by a standard argument with Lyapunov function (e.g., see Section 3.1 in [24]).

Proof of Lemma 3.8 Step 1. Let us write $u(t)=v(t)+z(t)$, where $z$ is a solution of (2.5) with $v_{0}=0$, i.e.,

$$
z=\sum_{d \in \mathbb{N}^{n}} \int_{0}^{t} e^{(t-\tau) \Delta} b_{d} \varphi_{d} \mathrm{~d} \beta_{d}^{\omega}
$$

Then

$$
\dot{v}-\Delta v+i|v+z|^{2}(v+z)=0, \quad v(0)=u_{0}
$$

Clearly for any $\delta \in(0,1]$ and $T>0$ we have

$$
\mathbb{P} \Omega_{\delta}>0, \quad \text { where } \Omega_{\delta}=\left\{\sup _{0 \leq t \leq T}|z(t)|_{\infty}<\delta\right\}
$$


Step 2. Due to (3.11),

$$
\dot{v}-\Delta v+i|v|^{2} v=L_{3}, \quad(t, x) \in Q_{T}=[0, T] \times K,
$$

where $L_{3}$ is a cubic polynomial in $v, \bar{v}, z, \bar{z}$ such that every its monomial contains $z$ or $\bar{z}$. Consider the function $r=|v(t, x)|$. Due to (3.12), for $\omega \in \Omega_{\delta}$ and outside the zero-set $X=\{r=0\} \subset Q_{T}$ the function $r$ satisfies the parabolic inequality

$$
\dot{r}-\Delta r \leq C \delta\left(r^{2}+1\right), \quad r(0, x)=|v(0, x)| \leq R+1 .
$$

Define $\tau=\inf \left\{t \in[0, T]:|r(t)|_{\infty} \geq R+2\right\}$, where $\tau=T$ if the set is empty. Then $\tau>0$ and for $0 \leq t \leq \tau$ the r.h.s. in (4.12) is $\leq C \delta\left((R+2)^{2}+1\right)=\delta C_{1}(R)$. Now consider the function

$$
\tilde{r}(t, x)=r-(R+1)-t \delta C_{1}(R) .
$$

Then $\tilde{r} \leq 0$ for $t=0$ and for $(t, x) \in \partial\left(Q_{T} \backslash K\right)$. Due to (4.12) and the definition of $\tau$, for $(t, x) \in Q_{\tau} \backslash X$ this function satisfies

$$
\dot{\tilde{r}}-\Delta \tilde{r} \leq C \delta\left(r^{2}+1\right)-\delta C_{1}(R) \leq 0
$$

Applying the maximum principle [17], we see that $\tilde{r} \leq 0$ in $Q_{\tau} \backslash K$. So for $t \leq \tau$ we have $r(t, x) \leq(R+1)+t \delta C_{1}(R)$. Choose $\delta$ so small that $T \delta C_{1}(R)<1$. Then $r(t, x)<R+2$ for $t \leq \tau$. So $\tau=T$ and we have proved that

$$
|v(t)|_{\infty}=|r(t)|_{\infty} \leq R+2 \quad \forall 0 \leq t \leq T \text { if } \delta \leq \delta(T, R), \omega \in \Omega_{\delta} .
$$

Step 3. It remains to estimate $\|v(t)\|$. To do this we first define $v_{1}(t, x)$ as a solution of Eq. (1.2) with $\eta=0$ and $v_{1}(0)=u_{0}$. Then

$$
\left\|v_{1}(t)\right\| \leq e^{-\alpha_{1} t}\left\|u_{0}\right\|, \quad\left|v_{1}(t)\right|_{\infty} \leq\left|u_{0}\right|_{\infty} \leq R
$$

since outside its zero-set the function $\left|v_{1}(t, x)\right|$ satisfies a parabolic inequality with the maximum principle (namely, Eq. (4.12) with $\delta=0$ ).

Step 4. Now we estimate $w=v-v_{1}$. This function solves the following equation:

$$
\dot{w}-\Delta w+i\left(|v+z|^{2}(v+z)-\left|v_{1}\right|^{2} v_{1}\right)=0, \quad w(0)=0 .
$$

Denoting $X=w+z$ (so that $v+z=X+v_{1}$ ), we see that the term in the brackets is a cubic polynomial $P_{3}$ of the variables $X, \bar{X}, v_{1}$ and $\bar{v}_{1}$, such that every its monomial contains $X$ or $\bar{X}$. Taking the $H$-scalar product of the $w$-equation with $w$ we get that

$$
\frac{1}{2} \frac{\mathrm{d}}{\mathrm{d} t}\|w\|^{2}+\|\nabla w\|^{2}=-\left\langle i P_{3}, w\right\rangle, \quad w(0)=0
$$


By (3.15), for $\omega \in \Omega_{\delta}$ the r.h.s. is bounded by $C^{\prime}(R, T)\left(\delta^{2}+\|w\|^{2}+\|w\|^{4}\right)$. Therefore

$$
\|w(T)\|^{2} \leq e^{2 C^{\prime \prime}(R, T)} \delta^{2}
$$

everywhere in $\Omega_{\delta}$, if $\delta$ is small.

Step 5. Since $u=w+v_{1}+z$, then by (3.15), (3.14) and (3.16), for every $\delta, T>0$ and for each $\omega \in \Omega_{\delta}$ we have

$$
\|u(T)\| \leq \delta+e^{-\alpha_{1} T} R+e^{C^{\prime \prime}(R, T) T} \delta=: \kappa .
$$

Since $u=v+z$, then $|u(T)|_{\infty} \leq \delta+R+2$. Choosing first $T \geq T(R, r)$ and next $\delta \leq \delta(R, r, T)$ we achieve $\kappa \leq r$. This proves the lemma with $L=R+3$.

\section{Ergodicity}

In this section, we analyse behaviour of the process $u(t)$ with respect to the norms $\|u\|$ and $|u|_{\infty}$ and next use an abstract theorem from [14] to prove that the process is mixing.

\subsection{Uniqueness of stationary measure and mixing}

First we recall the abstract theorem from [14] in the context of the CGL equation (1.2). Let us, as before, denote by $P_{t}(u, \Gamma)$ and $\mathfrak{P}_{t}^{*}$ the transition function and the family of Markov operators, associated with Eq. (1.2) in the space of Borel measures in $C_{0}(K)$. Let $u(t)$ be a trajectory of (1.2), starting from a point $u \in C_{0}(K)$. Let $u^{\prime}(t)$ be an independent copy of the process $u(t)$, starting from another point $u^{\prime}$, and defined on a probability space $\Omega^{\prime}$ which is a copy of $\Omega$. For a closed subset $G \subset C_{0}(K)$ we set $G^{2}=G \times G \subset C_{0}(K) \times C_{0}(K)$ and define the hitting time

$$
\boldsymbol{\tau}\left(G^{2}\right):=\inf \left\{t \geq 0: u(t) \in G, u^{\prime}(t) \in G\right\},
$$

which is a random variable on $\Omega \times \Omega^{\prime}$. The following result is an immediate consequence of Theorem 3.1.3 in [14].

Proposition 4.1 Let us assume that for any integer $m \geq 1$ there is a closed subset $G_{m} \subset C_{0}(K)$ and constants $\delta_{m}>0, T_{m} \geq 0$ such that $\delta_{m} \rightarrow 0$ as $m \rightarrow \infty$, and the following two properties hold:

(i) (recurrence) For any $u, u^{\prime} \in C_{0}(K), \boldsymbol{\tau}\left(G_{m}^{2}\right)<\infty$ almost surely.

(ii) (stability) For any $u, u^{\prime} \in G_{m}$

$$
\sup _{t \geq T_{m}}\left\|P_{t}(u, \cdot)-P_{t}\left(u^{\prime}, \cdot\right)\right\|_{\mathcal{L}\left(C_{0}(K)\right)}^{*} \leq \delta_{m} .
$$

Then the stationary measure $\mu$ of Eq. (1.2), constructed in Theorem 3.5, is unique and for any $\lambda \in \mathcal{P}\left(C_{0}(K)\right)$ we have $\mathfrak{P}_{t}^{*} \lambda \rightarrow \mu$ as $t \rightarrow \infty$. 
We will derive from this that the Markov process, defined by Eq. (1.2) in $C_{0}(K)$, is mixing:

Theorem 4.2 There is an integer $N=N\left(B_{*}\right) \geq 1$ such that if $b_{d} \neq 0$ for $|d| \leq N$, then there is a unique stationary measure $\mu \in \mathcal{P}\left(C_{0}(K)\right)$ for (1.2), and for any measure $\lambda \in \mathcal{P}\left(C_{0}(K)\right)$ we have $\mathfrak{P}_{t}^{*} \lambda \rightarrow \mu$ as $t \rightarrow \infty$.

The theorem is proved in the next section. Now we derive from it a corollary:

Corollary 4.3 Let $f(u)$ be a continuous functional on $C_{0}(K)$ such that $|f(u)| \leq$ $C_{f} e^{c|u|_{\infty}^{2}}$ for $u \in C_{0}(K)$, where $c<c_{*}\left(c_{*}>0\right.$ is the constant in assertion ( $\left.i\right)$ of Theorem 2.7). Then for any solution $u(t)$ of (1.2) such that $u(0) \in C_{0}(K)$ is nonrandom, we have

$$
\mathbb{E} f(u(t)) \rightarrow(\mu, f) \text { as } t \rightarrow \infty
$$

Proof For any $N \geq 1$ consider a smooth function $\varphi_{N}(r), 0 \leq \varphi_{N} \leq 1$, such that $\varphi_{N}=1$ for $|r| \leq N$ and $\varphi_{N}=0$ for $|r| \geq N+1$. Denote $f_{N}(u)=\varphi_{N}\left(|u|_{\infty}\right) f(u)$. Then $f_{N} \in C_{b}\left(C_{0}(K)\right)$, so by Theorem 4.2 we have

$$
\left|\mathbb{E} f_{N}(u(t))-\left(\mu, f_{N}\right)\right| \leq \kappa(N, t),
$$

where $\kappa \rightarrow 0$ as $t \rightarrow \infty$, for any $N$. Denote $v^{t}(d r)=\mathcal{D}\left(|u(t)|_{\infty}\right), t \geq 0$. Due to (2.18),

$$
\begin{aligned}
\mid \mathbb{E}\left(f_{N}(u(t))-f(u(t)) \mid\right. & \leq C_{f} \int_{0}^{\infty}\left(1-\varphi_{N}(r)\right) e^{c r^{2}} v^{t}(d r) \\
& \leq C_{f} e^{\left(c-c_{*}\right) N^{2}} \int_{0}^{\infty} e^{c_{*} r^{2}} v^{t}(d r) \leq C_{1} e^{\left(c-c_{*}\right) N}
\end{aligned}
$$

(note that the r.h.s. goes to 0 when $N$ grows to infinity). Similar, using Theorem 3.5 we find that $\left|\left(\mu, f_{N}\right)-(\mu, f)\right| \rightarrow 0$ as $N \rightarrow \infty$. The established relations imply the claimed convergence.

\subsection{Proof of Theorem 4.2}

It remains to check that eq. (1.2) satisfies properties (i) and (ii) in Proposition 4.1 for suitable sets $G_{m}$. For $m \in \mathbb{N}$ and $L>0$ we define

$$
G_{m, L}:=\left\{u \in C_{0}(K):\|u\| \leq \frac{1}{m},|u|_{\infty} \leq L\right\}
$$

(these are closed subsets of $C_{0}(K)$ ). For $u_{0}, u_{0}^{\prime} \in G_{m, L}$ consider solutions

$$
u=u\left(t, u_{0}\right), \quad u^{\prime}=u\left(t, u_{0}^{\prime}\right)
$$


defined on two independent copies $\Omega, \Omega^{\prime}$ of the probability space $\Omega$, and consider the first hitting time $\boldsymbol{\tau}\left(G_{m, L}^{2}\right)$ of the set $G_{m, L}^{2}$ by the pair $\left(u(t), u^{\prime}(t)\right)$ (this is a random variable on $\Omega \times \Omega^{\prime}$, see (4.1)). The proof of the following lemma is identical to that of Lemma 3.6.

Lemma 4.4 There is a constant $L^{\prime}>0$ such that for any $m \in \mathbb{N}$ we have

$$
\mathbb{E} e^{\gamma \tau\left(G_{m, L^{\prime}}^{2}\right)} \leq C\left(1+\left|u_{0}\right|_{\infty}^{2}+\left|u_{0}^{\prime}\right|_{\infty}^{2}\right) \text { for all } u_{0}, u_{0}^{\prime} \in C_{0}(K)
$$

where $\gamma$ and $C$ are suitable positive constants.

Let us choose $L=L^{\prime}$ in the definition of the sets $G_{m, L}$ in Proposition 4.1. Then the property (i) holds and it remains to establish (ii), where $P_{t}\left(u_{0}, \cdot\right)=\mathcal{D}(u(t))$ and $P_{t}\left(u_{0}^{\prime}, \cdot\right)=\mathcal{D}\left(u^{\prime}(t)\right)$. From now on we assume that the solutions $u$ and $u^{\prime}$ are defined on the same probability space. It turns out that it suffices to prove (4.2) with the norm $\|\cdot\|_{\mathcal{L}\left(C_{0}(K)\right)}^{*}$ replaced by $\|\cdot\|_{\mathcal{L}(H)}^{*}$. To show this we first estimate the distance between $\mathcal{D}(u(t))$ and $\mathcal{D}\left(u^{\prime}(t)\right)$ in the Kantorovich metrics

$$
\left\|\mathcal{D}(u(t))-\mathcal{D}\left(u^{\prime}(t)\right)\right\|_{K(H)}=\sup \left\{\left|(f, \mathcal{D}(u(t)))-\left(f, \mathcal{D}\left(u^{\prime}(t)\right)\right)\right|: \operatorname{Lip}(f) \leq 1\right\}
$$

in terms of

$$
d=\left\|\mathcal{D}(u(t))-\mathcal{D}\left(u^{\prime}(t)\right)\right\|_{\mathcal{L}(H)}^{*},
$$

where $t \geq 0$ is any fixed number. Without loss of generality, we can assume that the supremum in the definition of the Kantorovich distance is taken over $f \in \mathcal{L}(H)$ such that $\operatorname{Lip}(f) \leq 1$ and $f(0)=0$. By (2.18),

$$
\mathbb{E}\left(e^{c\|u(t)\|}+e^{c\left\|u^{\prime}(t)\right\|}\right) \leq C_{L} .
$$

Setting $f_{R}(u)=\min \{f(u), R\}$ and using (4.3), the Cauchy-Schwarz and Chebyshev inequalities, we get

$$
\mathbb{E}\left|f(u(t))-f_{R}(u(t))\right| \leq \mathbb{E}(\|u(t)\|-R) I_{\|u(t)\| \geq R} \leq C_{L}^{\prime} e^{-\frac{c}{2} R}
$$

A similar inequality holds for $u^{\prime}(t)$. Since $\left\|f_{R}\right\|_{\mathcal{L}(H)} \leq R+1$, then

$$
\mathbb{E}\left|f(u(t))-f\left(u^{\prime}(t)\right)\right| \leq 2 C_{L}^{\prime} e^{-\frac{c}{2} R}+(R+1) d .
$$

Optimising this relation in $R$, we find that $\mathbb{E}\left|f(u(t))-f\left(u^{\prime}(t)\right)\right| \leq C_{L}^{\prime \prime} \sqrt{d}$. Thus

$$
\left\|\mathcal{D}(u(t))-\mathcal{D}\left(u^{\prime}(t)\right)\right\|_{K(H)} \leq C_{L}^{\prime \prime} \sqrt{d},
$$

By (3.2), the functions $u(t)$ and $u^{\prime}(t)$ belong to $C^{\theta}(K)$ for any $\theta \in(0,1)$. The following interpolation inequality is proved at the end of this section. 
Lemma 4.5 For any $u \in C^{\theta}(K)$ we have

$$
|u|_{\infty} \leq C_{n, \theta}\|u\| \frac{2 \theta}{n+2 \theta}|u|_{C^{\theta}}^{\frac{n}{n+2 \theta}}
$$

By the celebrated Kantorovich theorem (e.g. see in [5]), we can find random variables $\xi$ and $\xi^{\prime}$ such that $\mathcal{D}(\xi)=\mathcal{D}(u(t)), \mathcal{D}\left(\xi^{\prime}\right)=\mathcal{D}\left(u^{\prime}(t)\right)$ and

$$
\mathbb{E}\left\|\xi-\xi^{\prime}\right\|=\left\|\mathcal{D}(u(t))-\mathcal{D}\left(u^{\prime}(t)\right)\right\|_{K(H)} \leq C_{L}^{\prime \prime} \sqrt{d}
$$

Using (4.4), (3.2), this estimate and the Hölder inequality, we find that

$$
\mathbb{E}\left|\xi-\xi^{\prime}\right|_{\infty} \leq C \mathbb{E}\left\|\xi-\xi^{\prime}\right\|^{\frac{2 \theta}{n+2 \theta}}\left|\xi-\xi^{\prime}\right|_{C^{\theta}}^{\frac{n}{n+2 \theta}} \leq\left(C_{L}^{\prime \prime} \sqrt{d}\right)^{\frac{2 \theta}{n+2 \theta}} C_{L}^{\prime \prime \prime} \frac{n}{n+2 \theta}=\tilde{C}_{L} d^{\frac{\theta}{n+2 \theta}}
$$

Therefore, for any $f$ such that $\|f\|_{\mathcal{L}\left(C_{0}(K)\right)} \leq 1$ we have

$$
\left|(f, \mathcal{D}(u(t)))-\left(f, \mathcal{D}\left(u^{\prime}(t)\right)\right)\right|=\left|\mathbb{E} f(\xi)-f\left(\xi^{\prime}\right)\right| \leq \mathbb{E}\left|\xi-\xi^{\prime}\right|_{\infty} \leq \tilde{C}_{L} d^{\frac{\theta}{n+2 \theta}}
$$

which implies that

$$
\left\|\mathcal{D}(u(t))-\mathcal{D}\left(u^{\prime}(t)\right)\right\|_{\mathcal{L}\left(C_{0}(K)\right)}^{*} \leq \tilde{C}_{L}\left(\left\|\mathcal{D}(u(t))-\mathcal{D}\left(u^{\prime}(t)\right)\right\|_{\mathcal{L}(H)}^{*}\right)^{\frac{\theta}{n+2 \theta}}
$$

Thus we have proved

Lemma 4.6 Assume that

$$
\sup _{t \geq T_{m}}\left\|P_{t}\left(u_{0}, \cdot\right)-P_{t}\left(u_{0}^{\prime}, \cdot\right)\right\|_{\mathcal{L}(H)}^{*} \leq \delta_{m}
$$

for all $u_{0}, u_{0}^{\prime} \in G_{m, L}$, where $\delta_{m} \rightarrow 0$. Then (4.2) holds for $G_{m}=G_{m, L}$ with $\delta_{m}^{\prime}=C_{L} \delta_{m}^{\frac{\theta}{n+2 \theta}}$.

So to prove Theorem 4.2 it remains to verify (4.6).

Proof of (4.6) In view of the triangle inequality we may assume that in (4.6) $u_{0}^{\prime}=0$.

Step 1. In this step we prove that it suffices to establish (4.6) for solutions of an equation, obtained by truncating the nonlinearity in (1.2). For any $\rho \geq 0$ and any continuous process $\{z(t): t \geq 0\}$ with range in $C_{0}(K)$ we define the stopping time

$$
\tau^{z}=\inf \left\{t \geq 0: \int_{0}^{t}|z(\tau)|_{\infty}^{2} d \tau-K t \geq \rho\right\},
$$

where $K$ is the constant in Lemma 2.8 (as usual, inf $\emptyset=\infty$ ). We set $\Omega_{\rho}^{z}=\left\{\tau^{z}<\infty\right\}$ and $\pi^{z}=\mathbb{P}\left(\Omega_{\rho}^{z}\right)$. Then 


$$
\pi^{u} \leq C e^{-\gamma \rho}, \quad \pi^{u^{\prime}} \leq C e^{-\gamma \rho}
$$

for suitable $C, \gamma>0$ and for any $\rho>0$. Consider the following auxiliary equation:

$$
\dot{v}-\Delta v+i|v|^{2} v+\lambda P_{N}(v-u)=\eta(t, x), \quad v(0)=0 .
$$

Consider $\tau^{v}$ and define $\Omega_{\rho}^{v}$ and $\pi^{v}$ as above. Define the stopping time

$$
\tau=\min \left\{\tau^{u}, \tau^{u^{\prime}}, \tau^{v}\right\} \leq \infty
$$

and define the continuous processes $\hat{u}(t), \hat{u}^{\prime}(t)$ and $\hat{v}(t)$ as follows: for $t \leq \tau$ they coincide with the processes $u, u^{\prime}$ and $v$ respectively, while for $t \geq \tau$ they satisfy the heat equation

$$
\dot{z}-\Delta z=\eta
$$

Due to (4.7)

$$
\|\mathcal{D}(u(t))-\mathcal{D}(\hat{u}(t))\|_{\mathcal{L}}^{*}+\left\|\mathcal{D}\left(u^{\prime}(t)\right)-\mathcal{D}\left(\hat{u}^{\prime}(t)\right)\right\|_{\mathcal{L}}^{*} \leq 4 \mathbb{P}\{\tau<\infty\} \leq 8 C e^{-\gamma \rho}+4 \pi^{v} .
$$

So to estimate the distance between $\mathcal{D}(u(t))$ and $\mathcal{D}\left(u^{\prime}(t)\right)$ it suffices to estimate $\pi^{v}$ and the distance between $\mathcal{D}(\hat{u}(t))$ and $\mathcal{D}\left(\hat{u}^{\prime}(t)\right)$.

Step 2. Let us first estimate the distance between $\mathcal{D}(\hat{u}(t))$ and $\mathcal{D}(\hat{v}(t))$. Equations (1.2) and (4.8) imply that for $t \leq \tau$ the difference $w=\hat{v}-\hat{u}$ satisfies

$$
\dot{w}-\Delta w+i\left(|\hat{v}|^{2} \hat{v}-|\hat{u}|^{2} \hat{u}\right)+\lambda P_{N} w=0, \quad w(0)=-u_{0},
$$

where $\left|\left\langle|\hat{v}|^{2} \hat{v}-|\hat{u}|^{2} \hat{u}, w\right\rangle\right| \leq C\left(|\hat{u}|_{\infty}^{2}+|\hat{v}|_{\infty}^{2}\right)\|w\|^{2}$. Taking the $H$-scalar product of the $w$-equation with $2 w$, we get that

$$
\frac{\mathrm{d}}{\mathrm{d} t}\|w\|^{2}+2\|\nabla w\|^{2}+2 \lambda\left\|P_{N} w\right\|^{2} \leq C\left(|\hat{u}|_{\infty}^{2}+|v|_{\infty}^{2}\right)\|w\|^{2}, \quad t \leq \tau .
$$

Since $\|\nabla w\|^{2} \geq \alpha_{N}\left\|Q_{N} w\right\|^{2}$, where $Q_{N}=\mathrm{id}-\mathrm{P}_{\mathrm{N}}$, then

$$
2\|\nabla w\|^{2}+2 \lambda\left\|P_{N} w\right\|^{2} \geq 2 \lambda_{1}\|w\|^{2}, \quad \lambda_{1}:=\min \left\{\alpha_{N}, \lambda\right\} .
$$

Choosing $\lambda$ and $N$ so large that $\lambda_{1}-C K \geq 1$ and applying to (4.10) the Gronwall inequality, we obtain that

$$
\begin{aligned}
\|w\|^{2} & \leq\left\|u_{0}\right\|^{2} \exp \left(-2 \lambda_{1} t+C \int_{0}^{t}\left(|\hat{u}|_{\infty}^{2}+|\hat{v}|_{\infty}^{2}\right) \mathrm{d} s\right) \\
& \leq \frac{1}{m^{2}} \exp \left(-2\left(\lambda_{1}-C K\right) t+2 C \rho\right) \leq \frac{1}{m^{2}} \exp (-2 t+2 C \rho),
\end{aligned}
$$


for $t \leq \tau$. Clearly for $t \geq \tau$ we have $(d / d t)\|w\|^{2} \leq-2\|w\|^{2}$. Therefore

$$
\|w\|^{2} \leq \frac{1}{m^{2}} \exp (-2 t+2 C \rho) \quad \forall t \geq 0 \quad \text { a.s. }
$$

So for any $f \in \mathcal{L}(H)$ such that $\|f\|_{\mathcal{L}} \leq 1$ we get

$$
|\mathbb{E}(f(\hat{u}(t))-f(\hat{v}(t)))| \leq\left(\mathbb{E}\|w\|^{2}\right)^{\frac{1}{2}} \leq \frac{1}{m} e^{C \rho-t}=: d(m, \rho, t) .
$$

Thus

$$
\|\mathcal{D}(\hat{u}(t))-\mathcal{D}(\hat{v}(t))\|_{\mathcal{L}(H)}^{*} \leq d(m, \rho, t)
$$

Step 3. To estimate the distance between $\mathcal{D}(\hat{v}(t))$ and $\mathcal{D}\left(\hat{u}^{\prime}(t)\right)$ notice that, without loss of generality, we can assume that the underlying probability space $(\Omega, \mathcal{F}, \mathbb{P})$ is of the particular form: $\Omega$ is the space of functions $u \in C\left(\mathbb{R}_{+}, C_{0}(K)\right)$ that vanish at $t=0, \mathbb{P}$ is the law of $\zeta$ defined by (1.3), and $\mathcal{F}$ is the completion of the Borel $\sigma$-algebra of $\Omega$ with respect to $\mathbb{P}$. For any $\omega . \in \Omega$, define the mapping $\Phi: \Omega \rightarrow \Omega$ by

$$
\Phi(\omega)_{t}=\omega_{t}-\lambda \int_{0}^{t} \chi_{s \leq \tau} P_{N}(\hat{v}(s)-\hat{u}(s)) \mathrm{d} s .
$$

Clearly, a.s. we have

$$
\hat{u}^{\prime \Phi(\omega)}(t)=\hat{v}^{\omega}(t) \quad \text { for all } t \geq 0
$$

Note that the transformation $\Phi$ is finite dimensional: it changes only the first $N$ components of a trajectory $\omega_{t}$. Due to (4.11), almost surely

$$
\int_{0}^{\infty}\left\|P_{N} w(s)\right\|^{2} d s \leq \frac{1}{2 m^{2}} e^{2 C \rho}
$$

This relation, the hypothesis that $b_{d} \neq 0$ for any $|d| \leq N$, and the argument in Section 3.3.3 of [14], based on the Girsanov theorem, show that

$$
\|\Phi \circ \mathbb{P}-\mathbb{P}\|_{v a r} \leq \frac{C(\rho)}{m}=: \tilde{d}(m, \rho) .
$$

Using (4.13), we get $\mathcal{D}(\hat{v}(t))=\hat{v}_{t} \circ \mathbb{P}=\hat{u}_{t}^{\prime} \circ(\Phi \circ \mathbb{P})$, where $\hat{v}_{t}$ stands for the random variable $\omega \rightarrow \hat{v}^{\omega}(t)$. Therefore,

$$
\begin{aligned}
\left\|\mathcal{D}(\hat{v}(t))-\mathcal{D}\left(\hat{u}^{\prime}(t)\right)\right\|_{\mathcal{L}(H)}^{*} & \leq 2\left\|\mathcal{D}(\hat{v}(t))-\mathcal{D}\left(\hat{u}^{\prime}(t)\right)\right\|_{\text {var }} \\
& \leq 2\|\Phi \circ \mathbb{P}-\mathbb{P}\|_{\text {var }} \leq 2 \tilde{d}(m, \rho)
\end{aligned}
$$


Step 4. Now let us prove (4.6). We get from (4.7) and (4.14) that

$$
\pi^{v}=\mathbb{P} \Omega_{\rho}^{v}=\mathbb{P} \Phi^{-1}\left(\Omega_{\rho}^{\hat{u}}\right)=(\Phi \circ \mathbb{P}) \Omega_{\rho}^{\hat{u}} \leq \mathbb{P} \Omega_{\rho}^{\hat{u}}+\tilde{d}(m, \rho) \leq C e^{-\gamma \rho}+\tilde{d}(m, \rho) .
$$

Due to (4.9), (4.12), (4.15) and the last inequality we have

$$
\begin{aligned}
\left\|\mathcal{D}(u(t))-\mathcal{D}\left(u^{\prime}(t)\right)\right\|_{\mathcal{L}}^{*} & \leq 12 C e^{-\gamma \rho}+d(m, \rho, t)+6 \tilde{d}(m, \rho) \\
& \leq 12 C e^{-\gamma \rho}+\frac{1}{m} e^{C \rho-t}+\frac{6}{m} C(\rho)=: D_{m}(t) .
\end{aligned}
$$

Let us choose $\rho=\rho(m)$, where $\rho(m) \rightarrow \infty$ in such a way that $\frac{6}{m} C(\rho(m)) \rightarrow 0$, and next take $T_{m}=C \rho(m)$. Then for $t \geq T_{m}$ we have $D_{m}(t) \leq \delta_{m} \rightarrow 0$. This completes the proof.

Proof of Lemma 4.5 Let us take any $u \in C^{\theta}, u \neq \equiv 0$ and set $M:=|u|_{\infty}, U:=|u|_{C^{\theta}}$. Take any $x_{*} \in K$ such that $\left|u\left(x_{*}\right)\right|=M$. To simplify the notation, we suppose that $x_{*}=0$. Regarding $u$ as an odd periodic function on $\mathbb{R}^{n}$ we have

$$
|u(x)| \geq M-|x|^{\theta} U \quad \forall x .
$$

The 1.h.s of this inequality vanishes at $|x|=(M / U)^{1 / \theta}=: r_{*} \leq 1$. Integrating the squared relation we get

$$
\begin{aligned}
\|u\|^{2} & \geq C \int_{0}^{r_{*}}\left(M-r^{\theta} U\right)^{2} r^{n-1} \mathrm{~d} r \\
& =C U^{2} \int_{0}^{r_{*}}\left(r_{*}^{2 \theta} r^{n-1}-2 r_{*}^{\theta} r^{n+\theta-1}+r^{n+2 \theta-1}\right) \mathrm{d} r \\
& =C U^{2} r_{*}^{n+2 \theta}\left(\frac{1}{n}-\frac{2}{n+\theta}+\frac{1}{n+2 \theta}\right)=U^{2} r_{*}^{n+2 \theta} C(n, \theta)>0 .
\end{aligned}
$$

Replacing in this inequality $r_{*}$ by its value we get (4.4).

\section{Some generalisations}

(1) Our proof, as well as that of [16], applies practically without any change to equations (1.1), where $v>0$ and $a \geq 0$. Indeed, scaling the time and $u$ we achieve $v=1$ (the random force scales to another force of the same type). Now consider Eq. (1.1) with $v=1$ and $a \geq 0$, and write the equation for $\xi(r(t, x))$. The integrand in the r.h.s. of Eq. (2.3) gets the extra term $-\xi^{\prime}(r) a r^{2}$. Accordingly, the r.h.s. part $g(t, x)$ of Eq. (2.8) gets the non-positive term $-a r^{2}$. Since the proof in Sect. 2 only uses that $g \leq \frac{1}{2 r} \sum b_{d}^{2}\left|\varphi_{d}\right|^{2}$, it does not change. In Sects. 3-4, as well as in [16], we only use results of Sect. 2 and the fact that the nonlinearity in the equation, as well as its derivatives up to order $m$, admit polynomial bounds. 
For the argument in Sect. 4 it is important that the nonlinearity's derivative grows no faster than $C|u|^{2}$.

(2) The proof of Theorem 2.2, given in [16], applies with minimal changes if the Sobolev space $H^{m}(K)$ with $m>n / 2$ (a Hilbert algebra) is replaced by the Sobolev space $W^{1, p}(K)$ with $p>n$ (a Banach algebra). It implies the assertions of the theorem with the norm $\|\cdot\|_{m}$ replaced by the norm $|\cdot|_{W^{1, p}}$, under the condition that $B_{1}<\infty$. The argument in Sects. 2.1-3.2 remains true in this setup since it does not use the $H^{m}$-norm. So to establish results of Sect. 3 one can use the $W^{1, p}$-solutions instead of $H^{m}$-solutions.

(3) Similar to (1) results of Sects. 2.1-3.2 remain true for Eq. (1.10).

(4) Consider Eq. (1.2) in a smooth bounded domain $\mathcal{O} \subset \mathbb{R}^{n}$ with Dirichlet boundary conditions:

$$
\left.u\right|_{\partial \mathcal{O}}=0 .
$$

Denote by $\left\{\varphi_{j}, j \geq 1\right\}$ the eigenbasis of $-\Delta$,

$$
-\Delta \varphi_{j}=\lambda_{j} \varphi_{j}, \quad j \geq 1
$$

and define the random field $\zeta(t, x)$ as in Sect. 1 , i.e. $\zeta=\sum_{j} b_{j} \beta_{j}(t) \varphi_{j}(x)$ Denote

$$
B_{*}=\sum_{j} b_{j}\left|\varphi_{j}\right|_{\infty}, \quad B_{1}=\sum_{j} b_{j}^{2}|\nabla \varphi|_{p}^{2}
$$

The $W^{1, p}$-argument as in (2) applies to Eq. (1.2), (5.1) and proves an analogy of Theorem 2.2 with the $\|\cdot\|_{m}$-norm replaced by the $|\cdot|_{W^{1, p}}$-norm, under the assumption that $B_{*}, B_{1}<\infty$. The only difference is that now the assertion of Lemma 2.4 follows not from [16], but from the result of [10] (also see [11,19]). After that the proof goes without any changes compare to Sects. 1-4 and establishes for Eqs. (1.2), (5.1) analogies of the main results of this work (with the space $C_{0}(K)$ replaced by $C_{0}(\mathcal{O})$ and $H^{1}$-by $H_{0}^{1}(\mathcal{O})$ ):

Theorem 5.1 Assume that $B_{*}<\infty$. Then

(i) for any $u_{0} \in C_{0}(\mathcal{O})$ problem (1.2), (1.6), (5.1) has a unique strong solution $u$ such that $u \in \mathcal{H}(0, \infty)$ a.s. This solution defines in the space $C_{0}(\mathcal{O})$ a Fellerian Markov process.

(ii) This process is mixing.

The first assertion remains true if in Eq. (1.2) we replace the nonlinearity by $i g_{r}\left(|u|^{2}\right) u, 0<r<\infty$. If $r \leq 1$, then the second assertion is also true. It is unknown if the systems, corresponding to equations with $r>1$, are mixing (this is a well known difficulty: it is unknown how to prove mixing for SPDEs without non-linear dissipation and with a conservative nonlinearity which grows at infinity faster then in the cubic way). 
(5) Lemmas 2.8, 4.4 and estimate (4.5) allow to apply to Eq. (1.2) the methods, developed recently to prove exponential mixing for the stochastic $2 \mathrm{~d}$ Navier-Stokes system (see in [14] Theorems 3.1.7, 3.4.1 as well as discussion of this result). It implies that the Markov process, defined by Eq. (1.2), is exponentially mixing, i.e. in Theorem 4.2 the distance $\left\|\mathfrak{P}_{t}^{*} \lambda-\mu\right\|_{\mathcal{L}}^{*}$ converges to zero exponentially fast. See Sect. 4 of [14] for consequences of this result. Proof of this generalization is less straightforward than those in (1-4) and will be presented elsewhere.

\section{Appendix. Proof of Lemma 2.5}

Let $v$ be a solution of the stochastic heat equation

$$
\dot{v}-\Delta v=\dot{\Upsilon}=\sum_{d \in \mathbb{N}^{n}} b_{d} f^{d}(t, x) \dot{\beta}_{d}(t), \quad v(0)=0,
$$

where $f^{d}(t, x)$ are progressively measurable functions such that $\left|f^{d}(t, x)\right| \leq L$ for each $d, t$ and $x$ almost surely, $b_{d}$ are real numbers satisfying (1.4), and $\beta_{d}$ are standard independent real-valued Brownian motions. By Lemma 2.4, we know that $v$ belongs to $C\left(\mathbb{R}_{+}, C_{0}(K)\right)$ a.s., and for any $t \geq 0$ and $p \geq 1$ estimate (2.7) holds. In this section we specify (2.7) and show that there is a constant $C(T)>0$ such that

$$
\mathbb{E} \sup _{\tau \in[t, t+T]}|v(\tau)|_{\infty}^{2 p} \leq\left(C(T) L B_{*}\right)^{2 p} p^{p}
$$

for all $t \geq 0$. To do this we reproduce the proof of Lemma 2.4, given in the Appendix to [16], tracing explicitly the values of the constants, involved in the estimates.

Step 1. Clearly it suffices to prove (6.2) for $T=1$. Moreover, it suffice to do this in the case when only one of the constants $b_{d}$ is non-zero. Indeed, let $v_{d}$ be the solution of (6.1) with $\dot{\Upsilon}=f^{d}(t, x) \dot{\beta}_{d}(t)$, and assume that we have

$$
\mathbb{E} \sup _{\tau \in[t, t+1]}\left|v_{d}(\tau)\right|_{\infty}^{2 p} \leq(C L)^{2 p} p^{p} \quad \forall d
$$

Then $v=\sum_{d \in \mathbb{N}^{n}} b_{d} v_{d}$, and the Minkovski inequality gives

$$
\begin{aligned}
\left(\mathbb{E} \sup _{\tau \in[t, t+1]}|v(\tau)|_{\infty}^{2 p}\right)^{1 / 2 p} & \leq\left(\mathbb{E}\left(\sum_{d} b_{d} \sup _{\tau \in[t, t+1]}\left|v_{d}\right|\right)^{2 p}\right)^{1 / 2 p} \\
& \leq \sum_{d} b_{d}\left(\mathbb{E} \sup _{\tau \in[t, t+1]}\left|v_{d}\right|^{2 p}\right)^{1 / 2 p} \leq B_{*} C L \sqrt{p},
\end{aligned}
$$

so we get (6.2).

Step 2 (estimates for increments). Let us write $v, f, \beta$ instead of $v_{d}, f^{d}, \beta_{d}$. At this step we show that for any $\theta \in(0,1 / 2)$ there is a constant $C(\theta)>0$ such that for any $t_{1}, t_{2} \in \mathbb{R}$ and $x_{1}, x_{2} \in \mathbb{R}^{n}$ with $\left|t_{1}-t_{2}\right| \leq 1$ and $\left|x_{1}-x_{2}\right| \leq 1$ we have 
$\mathbb{E}\left|v\left(t_{1}, x_{1}\right)-v\left(t_{2}, x_{2}\right)\right|^{p} \leq C(\theta)^{p} p^{\frac{p}{2}} L^{p}\left(\left|t_{1}-t_{2}\right|+\left|x_{1}-x_{2}\right|\right)^{\theta p}$,

for any $p>1$. Let us denote $g(t, \tau):=e^{(t-\tau) \Delta}\left(f\left(\tau, x_{1}\right)-f\left(\tau, x_{2}\right)\right)$ and

$$
U:=v\left(t, x_{1}\right)-v\left(t, x_{2}\right)=\int_{0}^{t} g(t, \tau) \mathrm{d} \beta(\tau)
$$

The quadratic variation of $U$ is given by $X(t):=\int_{0}^{t} g(t, \tau)^{2} \mathrm{~d} \tau$. Using the estimate

$$
\left\|e^{t \Delta} u\right\|_{C^{\theta}(K)} \leq C(\theta) t^{-\frac{\theta}{2}} e^{-c t}|u|_{\infty},
$$

valid for any $\theta \in(0,1)$ with suitable $c>0$ and $C(\theta)$ (e.g., see Lemma A1 in [16]), we get that

$$
X(t) \leq C(\theta) L^{2}\left|x_{1}-x_{2}\right|^{2 \theta} \int_{0}^{t} \tau^{-\theta} e^{-2 c \tau} \mathrm{d} \tau \leq C_{1}(\theta)\left|x_{1}-x_{2}\right|^{2 \theta} L^{2}
$$

Applying the Burkholder-Davis-Gundy (BDG) inequality (see [3]), we get

$$
\mathbb{E}|U|^{p} \leq C^{p} p^{\frac{p}{2}} \mathbb{E} X^{\frac{p}{2}} \leq C(\theta)^{p} L^{p} p^{\frac{p}{2}}\left|x_{1}-x_{2}\right|^{p \theta} .
$$

Now let us prove similar estimate for the time-increments. For any $\delta>0$ write $\delta$-time increment as

$$
\begin{aligned}
u(x, t+\delta)-u(x, t)= & \int_{t}^{t+\delta} e^{(t+\delta-\tau) \Delta} f(\tau, x) \mathrm{d} \beta(\tau) \\
& +\int_{0}^{t}\left(e^{(t+\delta-\tau) \Delta} f(\tau, x)-e^{(t-\tau) \Delta} f(\tau, x)\right) \mathrm{d} \beta(\tau) \\
& =: \int_{t}^{t+\delta} h_{1}(t, \tau) \mathrm{d} \beta(\tau)+\int_{0}^{t} h_{2}(t, \tau) \mathrm{d} \beta(\tau)=: I_{1}+I_{2} .
\end{aligned}
$$

If we show that

$$
\mathbb{E}\left|I_{1}\right|^{p} \leq C(\theta)^{p} L^{p} p^{\frac{p}{2}} \delta^{\frac{p}{2}}, \quad \mathbb{E}\left|I_{2}\right|^{p} \leq C(\theta)^{p} L^{p} p^{\frac{p}{2}} \delta^{\theta p},
$$

for any $\theta \in(0,1)$, then combining (6.5) with (6.6) we will get (6.4). But since the quadratic variations of $I_{1}$ and $I_{1}$ satisfy 


$$
\begin{aligned}
& \int_{t}^{t+\delta} h_{1}^{2}(t, \tau) \mathrm{d} \tau \leq L^{2} \delta, \\
& \int_{0}^{t} h_{2}^{2}(t, \tau) \mathrm{d} \tau \leq C(\theta) L^{2} \delta^{2 \theta} \int_{0}^{t} \tau^{-2 \theta} e^{-c \tau} \mathrm{d} \tau \leq C_{1}(\theta) L^{2} \delta^{2 \theta},
\end{aligned}
$$

then the BDG inequality implies (6.6) in the same way as above.

Step 3 (the Kolmogorov argument). Now we prove (6.3). To simplify calculations we scale $K$ to the unit cube, $K:=[0,1]^{n}$, and assume that $t=0$ (if not, we consider the function $\left.v^{\prime}\left(t^{\prime}, x\right)=v\left(t+t^{\prime}, x\right)\right)$. We specify $\theta=1 / 3$, denote $Q=[0,1] \times K=$ $[0,1]^{n+1}$ and define the sets

$$
\mathcal{K}_{N}=\left\{k \in \mathbb{Z}^{N+1}: k 2^{-N} \in Q\right\}, \quad N \geq 1
$$

For any $e=\left(e_{1}, \ldots, e_{n+1}\right) \in \mathbb{Z}^{n+1}$ such that $|e|=\max _{1 \leq j \leq n+1}\left|e_{j}\right|=1$, we set $\zeta_{k}^{N, e}=\left|v\left((k+e) 2^{-N}\right)-v\left(k 2^{-N}\right)\right|$. By Step 2 we have

$$
\mathbb{E}\left|\zeta_{k}^{N, e}\right|^{p} \leq C^{p} p^{\frac{p}{2}} L^{p} 2^{-p N / 3},
$$

for every $p>1$. For $q, R>0$ let us introduce the events

$$
\mathcal{A}_{k, q}^{N, e}=\left\{\omega \in \Omega: \zeta_{k}^{N, e} \geq R q^{N}\right\}, \quad \mathcal{A}_{q}^{N}=\cup_{k \in \mathcal{K}}\left(\cup_{|e|=1} \mathcal{A}_{k, q}^{N, e}\right) .
$$

From (6.7) and the Chebyshev inequality we get

$$
\mathbb{P}\left\{\mathcal{A}_{k, q}^{N, e}\right\} \leq R^{-p} q^{-p N} \mathbb{E}\left|\zeta_{k}^{N, e}\right|^{p} \leq C^{p} R^{-p} q^{-p N} p^{\frac{p}{2}} L^{p} 2^{-p N / 3}
$$

For each $N$ the total number of events $\mathcal{A}_{k, q}^{N, e}$ is not greater than $C^{\prime} 2^{(n+1) N}, C^{\prime}=C^{\prime}(n)$. Thus

$$
\mathbb{P}\left\{\mathcal{A}_{q}^{N}\right\} \leq C^{\prime} C^{p} R^{-p} q^{-p N} p^{\frac{p}{2}} L^{p} 2^{(n+1) N-p N / 3}=C^{\prime} C^{p} R^{-p} p^{\frac{p}{2}} L^{p} \alpha^{N},
$$

where $\alpha=q^{-p} 2^{(n+1)-p / 3}$. Let us choose $q=2^{-1 / 6}$ and $p \geq 6(n+2)$. Then $\alpha \leq 1 / 2$, and for the event $\mathcal{A}:=\cup_{N \geq 1} \mathcal{A}_{q}^{N}$ we have

$$
\mathbb{P}\{\mathcal{A}\} \leq C^{\prime} C^{p} R^{-p} p^{\frac{p}{2}} L^{p}
$$

Any point $x \in Q=[0,1]^{n+1}$ can be represented in the form $x=\sum_{j=1}^{\infty} e(j) 2^{-j}$, where $e(j) \in \mathbb{Z}^{n+1},|e(j)| \leq 1$. Let us set $x(0)=0$ and $x(m)=\sum_{j=1}^{m} e(j) 2^{-j}$ if $m \geq 1$. Then $v(t, x(0))=0$ for all $t \geq 0$, and for any $\omega \notin \mathcal{A}$

$$
|v(t, x(m))-v(t, x(m+1))| \leq R q^{m}=R 2^{-m / 6} .
$$


Therefore,

$$
|v(t, x)| \leq R \sum_{m=1}^{\infty} 2^{-m / 6}=R 2^{1 / 6}\left(2^{1 / 6}-1\right) .
$$

Combining this with (6.8), we get

$$
\mathbb{P}\left\{\|v\|_{L^{\infty}(Q)} \geq R\right\} \leq C_{1}^{p}(R+1)^{-p} p^{\frac{p}{2}} L^{p}
$$

for any $R>0$ and $p \geq 6(n+2)$. Thus for any $p$ like that we have

$$
\begin{aligned}
\mathbb{E}\|v\|_{L^{\infty}(Q)}^{p-1} & =\int_{0}^{\infty} x^{p-1} \mathrm{~d} \mathbb{P}\left\{\|v\|_{L^{\infty}(Q)} \leq x\right\}=(p-1) \int_{0}^{\infty} x^{p-2} \mathbb{P}\left\{\|v\|_{\left.L^{\infty}(Q) \geq x\right\} \mathrm{d} x}\right. \\
& \leq C_{1}^{p} p^{\frac{p}{2}} L^{p} \int_{0}^{\infty} x^{p-2}(x+1)^{-p} \mathrm{~d} x \leq C_{2}^{p} p^{\frac{p}{2}} L^{p},
\end{aligned}
$$

which implies (6.3) with a suitable $C$.

\section{References}

1. Brzezniak, Z., Gatarek, D.: Martingale solutions and invariant measures for stochastic evolution equations in Banach spaces. Stoch. Process. Appl. 84, 187-225 (1999)

2. Bogachev, V.I.: Gaussian Measures. American Mathematical Society, Providence (1998)

3. Burkholder, D.L.: Distribution function inequalities for martingales. Ann. Probab. 1(1), 19-42 (1973)

4. Debussche, A., Odasso, C.: Ergodicity for a weakly damped stochastic nonlinear Schrödinger equations. J. Evol. Eq. 3(5), 317-356 (2005)

5. Dudley, R.M.: Real Analysis and Probability. Cambrigde University Press, Cambridge (1989)

6. Da Prato, G., Zabczyk, J.: Stochastic Equations in Infinite Dimensions. Cambridge University Press, Cambridge (1992)

7. Da Prato, G., Zabczyk, J.: Ergodicity for Infinite-Dimensional Systems. Cambridge University Press, Cambridge (1996)

8. Hairer, M.: Exponential mixing properties of stochastic PDE's through asymptotic coupling. Probab. Theory Relat. Fields 124, 345-380 (2002)

9. Hairer, M., Mattingly, J.C.: A theory of hypoellipticity and unique ergodicity for semilinear stochastic PDEs. Electron. J. Probab. 16(23), 658-738 (2011)

10. Kuksin, S., Nadirashvili, N., Piatnitski, A.: Hölder estimates for solutions of parabolic SPDEs. Theory Probab. Appl. 47(1), 157-164 (2003)

11. Krylov, N.V.: On $L_{p}$-theory of stochastic partial differential equations in the wole space. SIAM J. Math. Anal. 27, 313-340 (1996)

12. Karatzas, I., Shreve, S.E.: Brownian Motion and Stochastic Calculus. Springer, New York (1991)

13. Kuksin, S., Shirikyan, A.: Coupling approach to white-forced nonlinear PDEs. J. Math. Pures Appl. 81, 567-602 (2002)

14. Kuksin, S., Shirikyan, A.: Mathematics of Two-Dimensional Turbulence. Cambridge University Press, Cambridge (2012)

15. Kuksin, S.: On turbulence in nonlinear Shrödinger equation. Geom. Funct. Anal. 7, 338-363 (1997)

16. Kuksin, S.: A stochastic nonlinear Schrödinger equation. I. A priori estimates. Tr. Mat. Inst. Steklova 225, 232-256 (1999) 
17. Landis, E.M.: Second Order Equations of Elliptic and Parabolic Type. American Mathematical Society, Providence (1997)

18. Lions, J.-L.: Quelques Méthodes de Résolution des Problèmes aux Limites Non Linéaires. GauthierVillars, Paris (1969)

19. Mikulevicius, R., Rozovskii, B.: A note on Krylov's $L_{p}$-theory for systems of SPDEs. Electron. J. Probab. 6(12), 1-35 (1996)

20. Nersesyan, V.: Polynomial mixing for the complex Ginzburg-Landau equation perturbed by a random force at random times. J. Evol. Eq. 8(1), 1-29 (2008)

21. Odasso, C.: Exponential mixing for stochastic PDEs: the non-additive case. Probab. Theory Relat. Fields 140, 41-82 (2008)

22. Shirikyan, A.: Exponential mixing for 2D Navier-Stokes equations perturbed by an unbounded noise. J. Math. Fluid Mech. 6(2), 169-193 (2004)

23. Shirikyan, A.: Ergodicity for a class of Markov processes and applications to randomly forced PDE's II. Discret. Contin. Dynam. Syst. 6(4), 911-926 (2006)

24. Shirikyan, A.: Exponential mixing for randomly forced PDE's: method of coupling. In: Bardos, C., Fursikov, A. (eds.) Instability of Models Connected with Fluid Flows II, pp. 155-188. Springer, New York (2008)

25. Vishik, M.I., Fursikov, A.V.: Mathematical Problems in Statistical Hydrodynamics. Kluwer, Dordrecht (1988) 